TRANSACTIONS OF THE

AMERICAN MATHEMATICAL SOCIETY

Volume 354, Number 2, Pages 491-535

S 0002-9947(01)02860-

Article electronically published on September 26, 2001

\title{
QUANTITATIVE ESTIMATES OF UNIQUE CONTINUATION FOR PARABOLIC EQUATIONS AND INVERSE INITIAL-BOUNDARY VALUE PROBLEMS WITH UNKNOWN BOUNDARIES
}

\author{
B. CANUTO, E. ROSSET, AND S. VESSELLA
}

\begin{abstract}
In this paper we obtain quantitative estimates of strong unique continuation for solutions to parabolic equations. We apply these results to prove stability estimates of logarithmic type for an inverse problem consisting in the determination of unknown portions of the boundary of a domain $\Omega$ in $\mathbb{R}^{n}$, from the knowledge of overdetermined boundary data for parabolic boundary value problems.
\end{abstract}

\section{INTRODUCTION}

In this paper we prove quantitative estimates of strong unique continuation for solutions to the parabolic equation

$$
u_{t}(x, t)-\operatorname{div}(\kappa(x) \nabla u(x, t))=0, \quad \text { in } \Omega \times(0, T],
$$

where $T$ is a positive number, $\Omega$ is a bounded domain in $\mathbb{R}^{n}, n \geq 3$, with sufficiently smooth boundary, $\kappa(x)=\left\{\kappa_{i j}(x)\right\}_{i, j=1}^{n}$ is a Lipschitz continuous matrix valued function satisfying a uniform ellipticity condition in $\Omega$ (see Part I, below). These estimates provide the main tools we use to obtain stability estimates for an inverse initial-boundary value problem concerning the determination of unknown boundaries (see Part II, below).

Part I (Quantitative Estimates of Unique Continuation for Parabolic Equations). We prove the following kinds of quantitative estimates of strong unique continuation for solutions to the parabolic equation (1.1):

a) Three spheres inequalities in the interior on the characteristic planes $t=t_{0}$, that is, roughly speaking,

$$
\left\|u\left(\cdot, t_{0}\right)\right\|_{L^{2}\left(\Delta_{\rho}\right)} \leq C\left\|u\left(\cdot, t_{0}\right)\right\|_{L^{2}\left(\Delta_{r}\right)}^{\frac{C}{\log (R / r)}}\left\|u\left(\cdot, t_{0}\right)\right\|_{L^{2}\left(\Delta_{R} \times(0, T)\right)}^{1-\frac{C}{\log (R / r)}},
$$

for every $t_{0} \in(0, T / 2)$ and $r<\rho<R$, where $\Delta_{s}$ denotes the open ball of radius $s$ and center at 0 in $\mathbb{R}^{n}$. See Theorems 3.1 .1 and $3.1 .1^{\prime}$ for a precise statement. The above inequality implies strong unique continuation on the

Received by the editors September 25, 2000.

2000 Mathematics Subject Classification. Primary 35R30; Secondary 35R25, 35R35.

Key words and phrases. Parabolic equations, strong unique continuation, stability, inverse problems.

Work supported in part by MURST. 
characteristic planes $t=t_{0}$ (see Corollary 3.1.7) and three cylinders inequalities in the interior (see Theorems 3.1.1 and 3.1.1').

b) Three spheres inequalities at the boundary on the characteristic planes $t=t_{0}$ and three cylinders inequalities at the boundary (see Theorems 3.2.1 and $\left.3.2 \cdot 1^{\prime}\right)$.

c) Stability estimates of continuation from Cauchy data on time-like surfaces (see Theorem 3.3.1).

d) Stability estimates of continuation from the interior (see Proposition 5.5).

The main tool which we use to derive all of these estimates is the so-called elliptic continuation of solutions to parabolic equations [Lan-O, which can be traced back to the pioneering work by Yamabe $[\mathbf{Y}$, who introduced this technique in 1959 to prove weak unique continuation properties for solutions to (1.1), when $\kappa \in C^{3}$ (see also [tY]).

Roughly speaking, the above mentioned elliptic continuation technique consists in the following idea: fixing $t_{0} \in(0, T)$, a solution $u(x, t)$ to the parabolic equation (1.1) can be continued for values of $y$, with $|y|<\delta$, in such a way that $u\left(x, t_{0}, y\right)$ satisfies an elliptic equation in $x$ and $y$. In this way, many properties of solutions of elliptic equations can be transferred to solutions of parabolic equations.

This technique was used again for parabolic equations of order $2 m$ by Landis and Oleinik [Lan-O] in 1974, to prove three spheres inequalities on the characteristic planes and strong unique continuation for solutions to (1.1) on these planes, under very strong regularity assumptions on $\kappa$, and, more recently, by $\mathrm{Lin} \mathrm{Li}$ in 1990 , to prove strong unique continuation for solutions to (1.1), assuming $\kappa$ Lipschitz continuous.

We exploit the above described elliptic continuation technique and quantitative estimates of strong unique continuation in the interior for elliptic equation (see [Lan, $[\mathrm{GL},[\mathrm{K}]$ ) to find estimates of type a).

Assuming that the boundary $\partial \Omega$ is of class $C^{1,1}$, we prove estimates of type b), by combining estimates of type a) with flattening of the boundary and reflection of the solution, elaborating on arguments contained in $\mathrm{AE}$.

In the elliptic continuation technique, analyticity properties with respect to $y$ of solutions to the elliptic equation

$$
\operatorname{div}(\kappa(x) \nabla w(x, y))+w_{y y}(x, y)=0,
$$

which is associated to (1.1) in the way described above, are used. We obtain these properties and the relative bounds on the derivatives of any order with respect to $y$ by adapting to (1.2) the Morrey-Nirenberg technique (see [M]). Furthermore, we need to find some stability estimates of continuation from Cauchy data on the plane $y=0$ for solutions to (1.2). We succeed in obtaining these estimates by exploiting hyperbolic continuation for solutions to (1.2), energy estimates and analytic continuation estimates.

Concerning estimates of type c), we recall that in [ Is1 and in [P] estimates of this kind are proved when $\kappa \in C^{1}$, see also [LavRS] (in the quoted papers $\kappa$ may also depend on $t$ ). When $\kappa$ only depends on $x$, we obtain the required estimates assuming that $\kappa$ is merely Lipschitz continuous, by using an extension in $H^{2}$ of the Cauchy data and the three spheres inequalities in the interior.

To obtain estimates of type d) we again combine estimates of type a) with the arguments used to prove Proposition 4.3 in [AlBRV]. 
Part II (Determining Boundaries in Inverse Conduction Problems). We deal with an inverse problem which arises in thermal imaging. This is a technique of nondestructive testing, to detect unknown corroded portions of the boundary or cavities in material objects, by measurements of temperature on an accessible portion of the surface. We refer to BryC1, BryC2 and references therein for more details and applications. More precisely, let $\Omega$ be a bounded domain in $\mathbb{R}^{n}$ with sufficiently smooth boundary $\partial \Omega$, and let us assume that an insulated portion $I$ of $\partial \Omega$ is not known. The thermal imaging technique consists in giving a heat flux input on an accessible portion $A$ of $\partial \Omega$ and measuring the temperature on $A$. Let us assume that $\kappa(x)=\left\{\kappa_{i j}(x)\right\}_{i, j=1}^{n}$ is the known symmetric thermal conductivity matrix, satisfying a uniform ellipticity condition, that $f$ is a known function on $\Omega$ and that $g$ is a nontrivial function on $\partial \Omega \times(0, T)$ such that $\operatorname{supp} g \subset A$. Then the temperature $u$ in $\Omega$ satisfies the following initial-boundary value problem of Neumann type:

$$
\begin{gathered}
u_{t}(x, t)-\operatorname{div}(\kappa(x) \nabla u(x, t))=0, \quad \text { in } \Omega \times(0, T], \\
u=f, \quad \text { on } \bar{\Omega} \times\{0\}, \\
\kappa \nabla u \cdot \nu=g, \quad \text { on } \partial \Omega \times[0, T],
\end{gathered}
$$

where $\nu$ denotes the outer unit normal to $\Omega$. It is well known that (1.3) has a unique solution.

Given an open subset $\Sigma$ of the boundary of $\Omega$ which is contained in $A$, we consider the inverse problem of determining $I$ from the knowledge of $u$ on $\Sigma \times[0, T]$.

We recall that uniqueness results for this problem have been proved by Isakov in [Is2] when $f \equiv 0, \kappa$ also depends on $t$, and other terms of lower order appear in equation (1.3a). In BryC1] Bryan and Caudill gave a counterexample showing that uniqueness may fail when $f \not \equiv 0$.

Let us also recall that in $[\mathrm{C}]$ the problem of determining $I$ from infinitely many measurements at a given time $t_{0}$ is studied.

In this paper we are mainly interested in studying the stability, that is, the continuous dependence of $I$ on the Cauchy data $u, \kappa \nabla u \cdot \nu$ on $\Sigma \times[0, T]$.

There is clear evidence that this inverse problem is severely ill-posed. In fact, in order to determine the unknown boundary $I$ it seems necessary to determine the interior values of $u$ from the Cauchy data on $\Sigma \times(0, T)$ up to $I$. Therefore it is reasonable to expect a weak rate of stability under a priori information on the unknown boundary portion $I$.

In 1997 Vessella $(\nabla)$ considered the case in which $\kappa \equiv I d$, the temperature is prescribed and the heat flux is measured. He proved logarithmic stability estimates, assuming that the prescribed temperature on $\partial \Omega$ is monotone with respect to time, by using analytic continuation techniques and properties of solutions to parabolic equations of constant sign.

Here, we prove logarithmic stability estimates under some a priori information on the domain, on $I$ and on the oscillation character of $g$. The proof of the stability result has the same structure as that in [AlBRV], and consists of two main steps. As a first step, we prove a rough estimate for the Hausdorff distance between the domains $\Omega_{1}, \Omega_{2}$ corresponding to the solutions $u_{1}, u_{2}$ whose boundary measurements are known with error, by using the estimates of type a)-d) described in Part I of this Introduction. As a second step, we employ in a more refined way the 
above mentioned estimates and a geometric lemma (Proposition 5.6), which has been proved in AlBRV, obtaining the logarithmic stability estimate.

Indeed, in the elliptic case, counterexamples by Alessandrini [Al] and Alessandrini and Rondi [AlR] show that logarithmic stability is best possible. This suggests that also in the parabolic case stability estimates better than logarithmic cannot be expected.

An interesting open problem is to find logarithmic stability estimates in the case in which $\kappa$ depends also on $t$. In fact, in this general case some estimates of unique continuation are available (see [Is1, [LeP], [P], LavRS]), but neither three spheres inequalities on the characteristic planes at the boundary nor three cylinders inequalities at the boundary are known. On the other side, these estimates at the boundary are the main tools we use in the proof of our stability result.

Connected to the inverse problem presented above is the question of uniqueness and stability when more general boundary conditions are prescribed on the unknown portion of the boundary, like, for instance,

$$
\kappa \nabla u \cdot \nu+\alpha u=0, \quad \text { on } \partial \Omega \times[0, T],
$$

where $\alpha \geq 0$ is a known function.

The plan of the paper is as follows.

In Section 2 we introduce some notation and definitions.

In Section 3, which we have subdivided into three subsections, we prove some quantitative estimates of strong unique continuation for solutions to (1.1), namely: three spheres inequalities in the interior (Theorem 3.1.1 and Theorem 3.1.1'), strong unique continuation on characteristic planes (Corollary 3.1.7), three spheres inequalities at the boundary (Theorem 3.2.1 and Theorem 3.2.1'), stability estimates of continuation from Cauchy data on time-like surfaces (Theorem 3.3.1), and the stability estimates of continuation from Cauchy data on the plane $y=0$ for solutions to (1.2) (Proposition 3.1.4).

In Section 4 we state the stability result for the inverse problem described in Part II (Theorem 4.1).

Section 5 contains the proof of Theorem 4.1, which is obtained through a sequence of Propositions. In particular, Proposition 5.5 provides estimates of type d).

Section 6 contains the proof of Propositions 5.1-5.5.

Finally, in Appendix A we have collected some interpolation and traces inequalities which we use throughout the paper.

\section{Notation And Definitions}

Let us introduce some notation.

We shall fix the space dimension $n \geq 3$ throughout the paper. Therefore we shall omit the dependence of the various quantities on $n$.

We shall use the letter $c$ to denote absolute constants, and the letters $C, \tilde{C}$ to denote constants depending on some a priori data. The value of the constants may change from line to line, but we have specified their dependence everywhere they appear.

We shall identify $\mathbb{R}^{2}$ and $\mathbb{C}$.

We shall denote by $B_{r}(a)\left(\Delta_{r}(a), \Delta_{r}^{\prime}(a), D_{r}(a)\right.$, respectively) the open ball in $\mathbb{R}^{n+1}\left(\mathbb{R}^{n}, \mathbb{R}^{n-1}, \mathbb{C}\right.$ respectively) centered at $a$, of radius $r$. Sometimes we shall write for brevity $B_{r}, \Delta_{r}, \Delta_{r}^{\prime}, D_{r}$ instead of $B_{r}(0), \Delta_{r}(0), \Delta_{r}^{\prime}(0), D_{r}(0)$, respectively. 
We shall denote $B_{r}^{+}=\left\{\left(x_{1}, \ldots, x_{n}, y\right) \in B_{r}\right.$ s.t. $\left.y>0\right\}, \Delta_{r}^{+}=\left\{\left(x_{1}, \ldots, x_{n}\right) \in\right.$ $\Delta_{r}$ s.t. $\left.x_{n}>0\right\}$. When dealing with $n+1$ variables $(x, \cdot)$, where $x=\left(x_{1}, \ldots, x_{n}\right)$, we shall denote $\nabla=\nabla_{x}$, $\operatorname{div}=\operatorname{div}_{x}, D^{2}=D_{x}^{2}$. Sometimes, for brevity, we shall write $\partial_{y}^{k} u$ instead of $\frac{\partial^{k} u}{\partial y^{k}}, u_{y}$ instead of $\frac{\partial u}{\partial y}$ and $u_{y y}$ instead of $\frac{\partial^{2} u}{\partial y^{2}}$.

Given an open set $\Omega \subset \mathbb{R}^{n}$ and $r>0$, we shall denote

$$
\Omega_{r}=\{x \in \Omega \text { s.t. } \operatorname{dist}(x, \partial \Omega)>r\} .
$$

When representing a boundary locally as a graph, it will be convenient to use the following notation. For every $x \in \mathbb{R}^{n}$ we shall set $x=\left(x^{\prime}, x_{n}\right)$, where $x^{\prime} \in \mathbb{R}^{n-1}$, $x_{n} \in \mathbb{R}$.

Definition 2.1. Let $\Omega$ be a bounded domain in $\mathbb{R}^{n}$. We shall say that a portion $S$ of $\partial \Omega$ is of class $C^{1,1}$ with constants $R_{0}, E>0$, if, for any $P \in S$, there exists a rigid transformation of coordinates under which we have $P=0$ and

$$
\Omega \cap \Delta_{R_{0}}=\left\{x \in \Delta_{R_{0}} \text { s.t. } x_{n}>\varphi\left(x^{\prime}\right)\right\},
$$

where $\varphi$ is a $C^{1,1}$ function on $\Delta_{R_{0}}^{\prime} \subset \mathbb{R}^{n-1}$ satisfying

$$
\varphi(0)=|\nabla \varphi(0)|=0
$$

and

$$
\|\varphi\|_{C^{1,1}\left(\Delta_{R_{0}}^{\prime}\right)} \leq E R_{0}
$$

Definition 2.2. Let $\Omega$ be a bounded domain in $\mathbb{R}^{n}$. We shall say that a portion $S$ of $\partial \Omega$ is of Lipschitz class with constants $R_{0}, E>0$, if, for any $P \in S$, there exists a rigid transformation of coordinates under which we have $P=0$ and

$$
\Omega \cap \Delta_{R_{0}}=\left\{x \in \Delta_{R_{0}} \text { s.t. } x_{n}>\varphi\left(x^{\prime}\right)\right\},
$$

where $\varphi$ is a Lipschitz continuous function on $\Delta_{R_{0}}^{\prime} \subset \mathbb{R}^{n-1}$ satisfying

$$
\varphi(0)=0
$$

and

$$
\|\varphi\|_{C^{0,1}\left(\Delta_{R_{0}}^{\prime}\right)} \leq E R_{0}
$$

Remark 2.1. We have chosen to normalize all norms in such a way that their terms are dimensionally homogeneous, and coincide with the standard definition when $R_{0}=1$ and $T=1$. For instance, for any $\varphi \in C^{1,1}\left(\Delta_{R_{0}}^{\prime}\right)$, we set

$$
\|\varphi\|_{C^{1,1}\left(\Delta_{R_{0}}^{\prime}\right)}=\|\varphi\|_{L^{\infty}\left(\Delta_{R_{0}}^{\prime}\right)}+R_{0}\|\nabla \varphi\|_{L^{\infty}\left(\Delta_{R_{0}}^{\prime}\right)}+R_{0}^{2}\left\|D^{2} \varphi\right\|_{L^{\infty}\left(\Delta_{R_{0}}^{\prime}\right)},
$$

and, for any $\varphi \in C^{0,1}\left(\Delta_{R_{0}}^{\prime}\right)$, we set

$$
\|\varphi\|_{C^{0,1}\left(\Delta_{R_{0}}^{\prime}\right)}=\|\varphi\|_{L^{\infty}\left(\Delta_{R_{0}}^{\prime}\right)}+R_{0}|\varphi|_{1, \Delta_{R_{0}}^{\prime}}
$$

Similarly, for any $u \in H^{2,1}(\Omega \times(0, T))$, we set

$$
\|u\|_{H^{2,1}(\Omega \times(0, T))}^{2}=\int_{\Omega \times(0, T)}\left(u^{2}+R_{0}^{2}|\nabla u|^{2}+R_{0}^{4}\left|D^{2} u\right|^{2}+T^{2} u_{t}^{2}\right) d x d t,
$$

and so on for boundary and trace norms such as $\|\cdot\|_{H^{3 / 2,3 / 4}\left(S_{T}\right)},\|\cdot\|_{H^{1 / 2,1 / 4}\left(S_{T}\right)}$, where we denote

$$
S_{T}=\partial \Omega \times(0, T) .
$$




\section{Quantitative estimates of StRong UniQue CONTINUATion}

We subdivide this section into three subsections.

In Subsection 3.1 we exploit the elliptic continuation technique to obtain three spheres inequalities in the interior and strong unique continuation on characteristic planes. First, we prove a form of the three spheres inequality in the interior suitable for the applications of Section 4, under the additional assumption that the solution vanishes at time $t=0$ (Theorem 3.1.1). Next, by a slight modification of the proof of Theorem 3.1.1, we prove the three spheres inequality in the interior also for solutions taking general initial data (Theorem 3.1.1'). As a consequence of Theorem 3.1.1', we derive strong unique continuation on characteristic planes (Corollary 3.1.7).

In Subsection 3.2, we prove the three spheres inequality at the boundary for solutions taking zero initial values (Theorem 3.2.1) and for solutions taking general initial values (Theorem 3.2.1').

In Subsection 3.3 we prove a stability result for the Cauchy problem for parabolic equations on time-like surfaces (Theorem 3.3.1).

Subsection 3.1 (Three Spheres Inequalities and Three Cylinders Inequalities in the Interior). We are given positive constants $T, R_{0}, R, \lambda, \lambda_{1}, \Lambda$ and $\Lambda_{1}$, satisfying $\lambda \geq 1, \lambda_{1} \geq 1,2 R \leq R_{0}$.

Let us denote by $c_{P}$ the absolute constant from the following Poincaré inequality:

$$
\int_{\Delta_{2 R}} f^{2}(x) d x \leq c_{P} R^{2} \int_{\Delta_{2 R}}|\nabla f(x)|^{2} d x, \quad \text { for every } f \in H_{0}^{1}\left(\Delta_{2 R}\right),
$$

where we recall that $c_{P} \leq 4$ (see [GT]) and that $c_{P}=\frac{4}{k_{0}^{2}}$, where $k_{0}$ is the smallest positive root of the Bessel function of first kind $J_{\frac{n-2}{2}}$ (see [CH]).

Let $\kappa(x)$ be a given function from $\Delta_{2 R}$, with values $n \times n$ symmetric matrices, satisfying the following conditions:

$$
\lambda^{-1}|\xi|^{2} \leq \kappa(x) \xi \cdot \xi \leq \lambda|\xi|^{2}, \quad \text { for every } x \in \Delta_{2 R} \text { and } \xi \in \mathbb{R}^{n}, \quad \text { (ellipticity) }
$$

$$
|\kappa(x)-\kappa(y)| \leq \frac{\Lambda}{R_{0}}|x-y|, \quad \text { for every } x, y \in \Delta_{2 R} . \quad \text { (Lipschitz continuity) }
$$

Let $b(x)$ be a given function from $\Delta_{2 R}$ satisfying the following conditions:

$$
\lambda_{1}^{-1} \leq b(x) \leq \lambda_{1}, \quad \text { for every } x \in \Delta_{2 R},
$$

$$
|b(x)-b(y)| \leq \frac{\Lambda_{1}}{R_{0}}|x-y|, \quad \text { for every } x, y \in \Delta_{2 R} \text {. }
$$

Let

$$
\begin{aligned}
& \lambda_{2}=\max \left\{\lambda, \lambda_{1}\right\}, \\
& \Lambda_{2}=\max \left\{\Lambda, \Lambda_{1}\right\} .
\end{aligned}
$$

Let $u(x, t) \in H^{2,1}\left(\Delta_{2 R} \times(0, T)\right)$ be a solution to the parabolic equation

$$
b(x) u_{t}(x, t)-\operatorname{div}(\kappa(x) \nabla u(x, t))=0, \quad \text { in } \Delta_{2 R} \times(0, T],
$$


satisfying

$$
u(\cdot, 0)=0, \quad \text { on } \Delta_{2 R} \times\{0\},
$$

and let

$$
H=\sup _{t \in[0, T]}\|u(\cdot, t)\|_{H^{1}\left(\Delta_{2 R}\right)}
$$

where

$$
\|u(\cdot, t)\|_{H^{1}\left(\Delta_{2 R}\right)}^{2}=\int_{\Delta_{2 R}}\left(u^{2}(x, t)+R_{0}^{2}|\nabla u(x, t)|^{2}\right) d x .
$$

Theorem 3.1.1. (Three Spheres Inequalities and Three Cylinders Inequalities in the Interior). Let $u \in H^{2,1}\left(\Delta_{2 R} \times(0, T)\right)$ be a solution to (3.1.5) and let (3.1.1) and (3.1.2) be satisfied. For every $r_{1}, r_{2}, r_{3}$ with $0<r_{1}<r_{2}<\left(4 \max \left\{\sqrt{2}, \lambda_{2}\right\}\right)^{-1} r_{3}$ $<r_{3} \leq \bar{\theta} R$, we have

$$
\int_{\Delta_{r_{2}}} u^{2}\left(x, t_{0}\right) d x
$$

$$
\begin{gathered}
\leq \tilde{C} \frac{r_{3}}{r_{3}-r_{2}}\left(\frac{r_{3}}{r_{2}}\right)^{C}\left(\left(1+\frac{T^{2}}{R^{4}}\right) H^{2}\right)^{1-\bar{\beta} \gamma^{\prime}}\left(\int_{\Delta_{r_{1}}} u^{2}\left(x, t_{0}\right) d x\right)^{\bar{\beta} \gamma^{\prime}}, \forall t_{0} \in\left(0, \frac{T}{2}\right), \\
\int_{0}^{\frac{T}{2}} \int_{\Delta_{r_{2}}} u^{2}(x, t) d x d t
\end{gathered}
$$

$$
\leq \tilde{C} \frac{r_{3}}{r_{3}-r_{2}}\left(\frac{r_{3}}{r_{2}}\right)^{C}\left(T\left(1+\frac{T^{2}}{R^{4}}\right) H^{2}\right)^{1-\bar{\beta} \gamma^{\prime}}\left(\int_{0}^{\frac{T}{2}} \int_{\Delta_{r_{1}}} u^{2}(x, t) d x d t\right)^{\bar{\beta} \gamma^{\prime}}
$$

where

$$
\begin{gathered}
\gamma^{\prime}=\frac{\log \left(\frac{1}{2}+\frac{r_{3}}{2 \lambda_{2} r_{2}^{\prime}}\right)}{\log \left(\frac{1}{2}+\frac{r_{3}}{2 \lambda_{2} r_{2}^{\prime}}\right)+C \log \frac{2 \lambda_{2} r_{2}^{\prime}}{a r_{1}}}, \\
r_{2}^{\prime}=\frac{4 \lambda_{2}-2}{4 \lambda_{2}-1} r_{2}+\frac{1}{4 \lambda_{2}-1} r_{3}, \\
a=\frac{3}{4 \sqrt{2} e \pi \lambda_{2}},
\end{gathered}
$$

$\bar{\beta}, 0<\bar{\beta}<1$, depends on $\lambda_{2}$ only, $\bar{\theta}, 0<\bar{\theta}<1$, and $C$ depend on $\lambda_{2}$ and $\Lambda_{2}$ only and $\tilde{C}$ depends on $\lambda_{2}, \Lambda_{2}$ and $\frac{R_{0}^{2}}{T}$ only.

Remark 3.1.2. $\quad$ i) Here, we study the more general equation (3.1.5a) instead of (1.1), since in our proof of the three spheres inequalities at the boundary (Theorem 3.2.1) we shall need the estimates (3.1.7) for solutions to equations of the form (3.1.5a) with a coefficient $b$ satisfying (3.1.2).

ii) Actually, a more correct terminology for inequality (3.1.7a) should be two spheres and one cylinder inequality (see the definition (3.1.6) of $H$ ), but we call it three spheres inequality for historical reasons (see [Lan-O]). 
iii) We have expressed the three cylinders inequality (3.1.7b) in terms of the $L^{\infty}-L^{2}$ norm over the cylinder $\Delta_{2 R} \times(0, T)$ (see the definition (3.1.6) of $H$ ). Let us notice, however, that also a more symmetric inequality, involving the $L^{2}$ norm over $\Delta_{2 R} \times(0, T)$, could be derived similarly.

In order to prove Theorem 3.1.1, we prove some preliminary lemmas concerning the technique of elliptic continuation (see also Li]) and a stability estimate of continuation from Cauchy data on the plane $y=0$ for solutions to the elliptic equation (1.2) (Proposition 3.1.4).

Let us start by fixing $t_{0} \in(0, T)$ and by considering the weak solution $\tilde{u}$ to the following initial-boundary value parabolic problem:

$$
\begin{gathered}
b(x) \tilde{u}_{t}(x, t)-\operatorname{div}(\kappa(x) \nabla \tilde{u}(x, t))=0, \quad \text { in } \Delta_{2 R} \times(0,+\infty), \\
\tilde{u}=h, \quad \text { on } \partial \Delta_{2 R} \times[0,+\infty), \\
\tilde{u}=0, \quad \text { on } \Delta_{2 R} \times\{0\},
\end{gathered}
$$

where $h$ is the extension by 0 of $\eta(t) u(x, t)$, and where $\eta \in C^{2}([0,1])$ is a cut-off function such that $\eta \equiv 1$ in $\left[0, t_{0}\right], \eta \equiv 0$ in $[T,+\infty)$ and $\left|\eta^{\prime}\right| \leq \frac{c}{T-t_{0}}$.

It is evident that

$$
\tilde{u}\left(\cdot, t_{0}\right)=u\left(\cdot, t_{0}\right), \quad \text { in } \Delta_{2 R}
$$

Let us denote

$$
\begin{gathered}
a_{R}=\left(\lambda \lambda_{1} c_{P} R^{2}\right)^{-1}, \\
q=\frac{T}{T-t_{0}} .
\end{gathered}
$$

Lemma 3.1.2. Let $u, \tilde{u}$ be as above. We have

$$
\|\tilde{u}(\cdot, t)\|_{H^{1}\left(\Delta_{2 R}\right)}^{2} \leq c C_{1}^{2} H^{2} \exp \left(-2 a_{R}(t-T)_{+}\right),
$$

where

$$
C_{1}^{2}=\lambda_{2}^{2} q\left(\frac{R_{0}^{2}}{T-t_{0}}+e^{c q}\right)
$$

Proof of Lemma 3.1.2. Let

$$
v=\tilde{u}-\eta u,
$$

where $\eta$ is the cut-off function introduced above. We have that $v$ satisfies

$$
\begin{gathered}
b(x) v_{t}(x, t)-\operatorname{div}(\kappa(x) \nabla v(x, t))=-b(x) \eta^{\prime}(t) u(x, t), \quad \text { in } \Delta_{2 R} \times(0, T], \\
v=0, \quad \text { on } \partial \Delta_{2 R} \times[0, T], \\
v=0, \quad \text { on } \Delta_{2 R} \times\{0\} .
\end{gathered}
$$

Multiplying equation (3.1.14a) by $v$ and integrating over $\Delta_{2 R} \times[0, t]$, we obtain, for $t \in[0, T]$,

$$
\int_{\Delta_{2 R}} b(x) v^{2}(x, t) d x \leq c \lambda_{1} q H^{2}+\left(\frac{c}{T-t_{0}}\right) \int_{0}^{t} d \tau \int_{\Delta_{2 R}} b(x) v^{2}(x, \tau) d x .
$$


By applying the Peano-Gronwall inequality to (3.1.15), we have

$$
\int_{\Delta_{2 R}} b(x) v^{2}(x, t) d x \leq c \lambda_{1} q e^{c q} H^{2} .
$$

Choosing $t=T$ in $(3.1 .16)$ and recalling that $v(\cdot, T)=\tilde{u}(\cdot, T)$, we obtain

$$
\int_{\Delta_{2 R}} b(x) \tilde{u}^{2}(x, T) d x \leq c \lambda_{1} q e^{c q} H^{2} .
$$

Similarly, multiplying equation (3.1.14a) by $v_{t}$ and integrating over $\Delta_{2 R} \times[0, t]$, we obtain, for $t \in[0, T]$,

$$
\begin{aligned}
& \int_{\Delta_{2 R}} \kappa(x) \nabla v(x, t) \cdot \nabla v(x, t) d x \leq c \lambda_{1} \frac{t}{\left(T-t_{0}\right)^{2}} H^{2}, \\
& \int_{\Delta_{2 R}} \kappa(x) \nabla \tilde{u}(x, T) \cdot \nabla \tilde{u}(x, T) d x \leq c \lambda_{1} \frac{T}{\left(T-t_{0}\right)^{2}} H^{2} .
\end{aligned}
$$

Let us denote by $\mu_{k}$, with $\mu_{1}>\mu_{2} \geq \ldots \geq \mu_{k} \geq \ldots$, the negative eigenvalues associated to the problem

$$
\frac{1}{b(x)} \operatorname{div}(\kappa(x) \nabla \varphi(x))=\mu \varphi(x), \quad \text { in } \Delta_{2 R},
$$

$$
\varphi=0, \quad \text { on } \partial \Delta_{2 R},
$$

and by $\varphi_{k}$ the corresponding eigenfunctions normalized by

$$
\int_{\Delta_{2 R}} b(x) \varphi_{k}^{2}(x) d x=1 .
$$

We have

$$
0>-a_{R} \geq \mu_{1}>\mu_{2} \geq \ldots \geq \mu_{k} \geq \ldots
$$

Since $\tilde{u}=0$ on $\partial \Delta_{2 R} \times[T,+\infty)$, we have

$$
\tilde{u}(x, t)=\sum_{k=1}^{\infty} \beta_{k} \varphi_{k}(x) e^{\mu_{k}(t-T)}, \quad \text { for every } t \geq T,
$$

where

$$
\beta_{k}=\int_{\Delta_{2 R}} b(x) \varphi_{k}(x) \tilde{u}(x, T) d x .
$$

Recalling (3.1.17), we have

$$
\begin{gathered}
\int_{\Delta_{2 R}} b(x) \tilde{u}^{2}(x, t) d x=\sum_{k=1}^{\infty} \beta_{k}^{2} e^{2 \mu_{k}(t-T)} \leq e^{-2 a_{R}(t-T)} \int_{\Delta_{2 R}} b(x) \tilde{u}^{2}(x, T) d x \\
\leq c \lambda_{1} q e^{c q} H^{2} e^{-2 a_{R}(t-T)}, \quad \text { for every } t \geq T .
\end{gathered}
$$

Moreover,

$$
\begin{aligned}
\int_{\Delta_{2 R}} \kappa(x) \nabla \tilde{u}(x, t) \cdot \nabla \tilde{u}(x, t) d x & =-\int_{\Delta_{2 R}} b(x) \tilde{u}(x, t) \tilde{u}_{t}(x, t) d x \\
& =\sum_{k=1}^{\infty}\left|\mu_{k}\right| \beta_{k}^{2} e^{2 \mu_{k}(t-T)}
\end{aligned}
$$


Choosing $t=T$ in (3.1.24) and using (3.1.19), we can estimate

$$
\sum_{k=1}^{\infty}\left|\mu_{k}\right| \beta_{k}^{2} \leq c \lambda_{1} \frac{T}{\left(T-t_{0}\right)^{2}} H^{2}
$$

From (3.1.24) and (3.1.25) we have

$$
R_{0}^{2} \int_{\Delta_{2 R}}|\nabla \tilde{u}(x, t)|^{2} d x \leq c \lambda \lambda_{1} \frac{T R_{0}^{2}}{\left(T-t_{0}\right)^{2}} H^{2} e^{-2 a_{R}(t-T)} \quad \text { for every } t \geq T .
$$

From (3.1.23) and (3.1.26) we obtain

$$
\|\tilde{u}(\cdot, t)\|_{H^{1}\left(\Delta_{2 R}\right)}^{2} \leq c \lambda_{1} \lambda_{2} q\left(e^{c q}+\frac{R_{0}^{2}}{\left(T-t_{0}\right)}\right) H^{2} e^{-2 a_{R}(t-T)} \quad \text { for every } t \geq T .
$$

On the other hand, from (3.1.16) and (3.1.18), recalling that $\tilde{u}=v+\eta u$, we have

$$
\|\tilde{u}(\cdot, t)\|_{H^{1}\left(\Delta_{2 R}\right)}^{2} \leq c \lambda_{1} \lambda_{2} q\left(e^{c q}+\frac{R_{0}^{2}}{\left(T-t_{0}\right)}\right) H^{2} \quad \text { for every } t \leq T,
$$

so that (3.1.12) follows from (3.1.27) and (3.1.28).

Let us still denote by $\tilde{u}$ the extension by 0 of $\tilde{u}$ to $\Delta_{2 R} \times \mathbb{R}$, and let us consider the Fourier transform of $\tilde{u}$ with respect to the variable $t$,

$$
\hat{u}(x, \mu)=\int_{-\infty}^{+\infty} e^{-\mu i t} \tilde{u}(x, t) d t=\int_{0}^{+\infty} e^{-\mu i t} \tilde{u}(x, t) d t, \quad \text { for every } \mu \in \mathbb{R} .
$$

We have that $\hat{u}$ satisfies

$$
b(x) i \mu \hat{u}(x, \mu)-\operatorname{div}(\kappa(x) \nabla \hat{u}(x, \mu))=0, \quad \text { in } \Delta_{2 R} \times \mathbb{R} .
$$

Lemma 3.1.3. Let $u, \tilde{u}, \hat{u}$ be as above. We have

$$
\|\hat{u}(\cdot, \mu)\|_{L^{2}\left(\Delta_{R}\right)} \leq c C_{1} \lambda_{2} H e^{-\sqrt{|\mu|} \delta}\left(T+\frac{1}{a_{R}}\right),
$$

where

$$
\delta=\frac{R}{4 e \pi \lambda_{2}} .
$$

Proof of Lemma 3.1.3. Let us denote, for every $\mu, \xi \in \mathbb{R}, x \in \Delta_{2 R}$,

$$
v(x, \xi ; \mu)=e^{i \sqrt{|\mu|} \xi} \hat{u}(x, \mu) .
$$

For every $\mu \in \mathbb{R} \backslash\{0\}$, the function $v=v(\cdot, \cdot ; \mu)$ solves the uniformly elliptic equation

$$
\operatorname{div}(\kappa \nabla v)+i b \operatorname{sgn}(\mu) \frac{\partial^{2} v}{\partial \xi^{2}}=0, \quad \text { in } \Delta_{2 R} \times \mathbb{R} .
$$

Let us denote $a_{j}=2-\frac{j}{k}$, for every $j \in\{0,1, \ldots, k\}$, and $k \in \mathbb{N}$. Moreover, let

$$
h_{j}(s)=\left\{\begin{array}{cc}
0 & \text { if }|s|>a_{j}, \\
\frac{1}{2}\left(1+\cos \left(\frac{\pi\left(a_{j+1}-s\right)}{a_{j+1}-a_{j}}\right)\right) & \text { if } a_{j+1} \leq|s| \leq a_{j}, \\
1 & \text { if }|s|<a_{j+1},
\end{array}\right.
$$


and

$$
v_{j}=\frac{\partial^{j} v}{\partial \xi^{j}}
$$

We have that $v_{j}$ solves the equation

$$
\operatorname{div}\left(\kappa \nabla v_{j}\right)+i b \operatorname{sgn}(\mu) \frac{\partial^{2} v_{j}}{\partial \xi^{2}}=0, \quad \text { in } \Delta_{2 R} \times \mathbb{R} .
$$

Multiplying equation (3.1.34) by $\bar{v}_{j} \eta_{j}^{2}$, where

$$
\eta_{j}=\eta_{j}(x, \xi)=h_{j}\left(\frac{|x|}{R}\right) h_{j}\left(\frac{\xi}{R}\right),
$$

and integrating over $D_{j}=\Delta_{a_{j} R} \times\left(-a_{j} R, a_{j} R\right)$, we obtain

$$
\begin{gathered}
\left(\int_{D_{j}} \kappa \nabla v_{j} \cdot \nabla \bar{v}_{j} \eta_{j}^{2} d x d \xi\right)^{2}+\left(\int_{D_{j}} b\left|\frac{\partial v_{j}}{\partial \xi}\right|^{2} \eta_{j}^{2} d x d \xi\right)^{2} \\
\leq \frac{8 \lambda_{2}^{2} \pi^{4} k^{4}}{R^{4}}\left(\int_{D_{j}}\left|v_{j}\right|^{2} d x d \xi\right)^{2}
\end{gathered}
$$

Therefore, for every $j \in\{0,1, \ldots, k\}$ we obtain

$$
\int_{D_{j+1}}\left|\frac{\partial^{j+1} v}{\partial \xi^{j+1}}\right|^{2} d x d \xi \leq \frac{\sqrt{8} \lambda_{1} \lambda_{2} \pi^{2} k^{2}}{R^{2}} \int_{D_{j}}\left|\frac{\partial^{j} v}{\partial \xi^{j}}\right|^{2} d x d \xi .
$$

By iteration of (3.1.36) for $j=0, \ldots, k-1$, we have

$$
\int_{\Delta_{R} \times(-R, R)}\left|\frac{\partial^{k} v}{\partial \xi^{k}}\right|^{2} d x d \xi \leq 4 R\left(\frac{\sqrt{8} \lambda_{1} \lambda_{2} \pi^{2} k^{2}}{R^{2}}\right)^{k} \int_{\Delta_{2 R}} \hat{u}^{2}(x, \mu) d x .
$$

Now, let us estimate the integral on the right hand side of (3.1.37). By (3.1.12), we obtain

$$
\|\hat{u}(\cdot, \mu)\|_{L^{2}\left(\Delta_{2 R}\right)} \leq \int_{0}^{+\infty}\|\tilde{u}(\cdot, t)\|_{L^{2}\left(\Delta_{2 R}\right)} d t \leq c C_{1} H\left(T+\frac{1}{a_{R}}\right) .
$$

Therefore, by (3.1.37), we have that, for every $\mu \in \mathbb{R} \backslash\{0\}$ and for every $k \in \mathbb{N}$,

$$
\int_{\Delta_{R} \times(-R, R)}\left|\frac{\partial^{k} v}{\partial \xi^{k}}\right|^{2} d x d \xi \leq c R\left(T+\frac{1}{a_{R}}\right)^{2} C_{1}^{2} H^{2}\left(\frac{\sqrt{8} \lambda_{1} \lambda_{2} \pi^{2} k^{2}}{R^{2}}\right)^{k} .
$$

For fixed $\mu \in \mathbb{R} \backslash\{0\}$ and $\varphi \in L^{2}\left(\Delta_{R}, \mathbb{C}\right)$, let us denote

$$
F(\xi)=\int_{\Delta_{R}} v(x, \xi ; \mu) \bar{\varphi}(x) d x .
$$

By the interpolation inequality (A.1) and by the inequality (3.1.39) we have

$$
\left|F^{(k)}(\xi)\right| \leq \frac{c C_{1} H}{R^{k}}\left(T+\frac{1}{a_{R}}\right)\left(2 \lambda_{2} \pi(k+1)\right)^{k+1}\|\varphi\|_{L^{2}\left(\Delta_{R}\right)} .
$$

By using inequality (3.1.40) for every $k \in \mathbb{N}$ and the power series of $F$ at any point $\xi_{0}$ such that $\Re e \xi_{0} \in(-R, R), \Im m \xi_{0}=0$, we have that the function $F$ can be analitycally extended to the rectangle $\{\xi \in \mathbb{C}$ s.t. $\Re e \xi \in(-R, R), \Im m \xi \in(-\bar{\rho}, \bar{\rho})\}$, 
where $\bar{\rho}=\frac{R}{2 e \pi \lambda_{2}}$. We continue to denote by $F$ the analytic extension of $F$. In particular, choosing $\xi_{0}=0$, we obtain the estimate

$$
|F(-i \delta)| \leq c C_{1} \lambda_{2} H\left(T+\frac{1}{a_{R}}\right)\|\varphi\|_{L^{2}\left(\Delta_{R}\right)},
$$

where $\delta$ is given by (3.1.32). On the other side, by the definition of $v$, we have

$$
F(-i \delta)=\int_{\Delta_{R}} e^{\sqrt{|\mu|} \delta} \hat{u}(x, \mu) \bar{\varphi}(x) d x,
$$

so that we obtain (3.1.31) from (3.1.41).

Estimate (3.1.31) allows us to define, for $x \in \Delta_{R},|y|<\sqrt{2} \delta$, the function

$$
w(x, y)=\frac{1}{2 \pi} \int_{-\infty}^{+\infty} e^{i t_{0} \mu} \hat{u}(x, \mu) \cosh \sqrt{-i \mu} y d \mu
$$

where

$$
\sqrt{-i \mu}=|\mu|^{\frac{1}{2}} e^{-\frac{\pi}{4} i \operatorname{sgn}(\mu)}
$$

We have that $w \in H_{l o c}^{1}\left(\Delta_{R} \times(-\sqrt{2} \delta, \sqrt{2} \delta)\right)$ is a solution to the following elliptic equation:

$$
\operatorname{div}(\kappa(x) \nabla w(x, y))+b(x) w_{y y}(x, y)=0, \quad \text { in } \Delta_{R} \times(-\sqrt{2} \delta,+\sqrt{2} \delta),
$$

and satisfies the following conditions:

$$
\begin{gathered}
w(x, 0)=u\left(x, t_{0}\right), \quad \text { in } \Delta_{R}, \\
w_{y}(x, 0)=0, \quad \text { in } \Delta_{R} .
\end{gathered}
$$

Let us notice that $w$ is even with respect to the $y$ variable.

Now, let us state and prove the following stability estimate for solutions to the Cauchy problem for elliptic equations.

Proposition 3.1.4. (Stability Estimates of Continuation from Cauchy Data on the Plane $y=0$ for Solutions to (1.2)). Let $L$ and $\rho$ be positive numbers such that $0<\rho<R$. Let $w$ be the solution to the following Cauchy problem:

$$
\begin{gathered}
\operatorname{div}(\kappa(x) \nabla w(x, y))+b(x) w_{y y}(x, y)=0, \quad \text { in } \Delta_{2 \rho} \times(-2 L, 2 L), \\
w(x, 0)=f(x), \quad \text { in } \Delta_{2 \rho}, \\
w_{y}(x, 0)=0, \quad \text { in } \Delta_{2 \rho},
\end{gathered}
$$

where $\kappa$ satisfies (3.1.1) and b satisfies (3.1.2). Let

$$
\begin{gathered}
\rho_{1}=\left(\pi e \lambda_{2}\left(\frac{1}{\rho^{2}}+\frac{1}{L^{2}}\right)^{1 / 2}\right)^{-1}, \\
\rho_{2}=\min \left\{\rho_{1}, \frac{\rho}{2 \sqrt{\lambda} \lambda_{1}}\right\}, \\
\rho_{3}=\frac{1}{2}\left(\rho-\sqrt{\lambda} \lambda_{1} \rho_{2}\right) .
\end{gathered}
$$


For every $y \in\left(-\frac{3}{8} \rho_{1}, \frac{3}{8} \rho_{1}\right)$ we have

$$
\begin{gathered}
\int_{\Delta_{\rho_{3}}}\left(w_{y}^{2}(x, y)+|\nabla w(x, y)|^{2}\right) d x \\
\leq C\left(\frac{1}{L \rho^{2}}\|w\|_{L^{2}\left(\Delta_{2 \rho} \times(-2 L, 2 L)\right)}^{2}+\|\nabla f\|_{L^{2}\left(\Delta_{\rho / 2}\right)}^{2}\right)^{1-\beta}\|\nabla f\|_{L^{2}\left(\Delta_{\rho / 2}\right)}^{2 \beta},
\end{gathered}
$$$$
\text { where } \beta, 0<\beta<1 \text {, depends on } \lambda_{2} \text { only and } C \text { depends on } \lambda_{2} \text { and } L \rho^{-1} \text { only. }
$$

Proof of Proposition 3.1.4 (Preparation). We preface the proof with two auxiliary steps.

Step 1. The power series

$$
\sum_{k=0}^{\infty} \frac{\partial^{k} w}{\partial y^{k}}(\cdot, 0) \frac{z^{k}}{k !}
$$

converges in $H^{2}\left(\Delta_{\frac{3}{4}} \rho\right)$ for every complex number $z$ such that $|z|<\rho_{1}$.

Proof of Step 1. Let us denote $Q_{0}=\Delta_{2 \rho} \times(-2 L, 2 L), Q_{1}=\Delta_{\rho} \times(-L, L)$. By a slight modification of the arguments used to prove (3.1.37), we obtain

$$
\left\|\partial_{y}^{k} w\right\|_{L^{2}\left(Q_{1}\right)}^{2} \leq\left(C_{1} k^{2}\right)^{k}\|w\|_{L^{2}\left(Q_{0}\right)}^{2} \quad \text { for every } k \geq 1
$$

where

$$
C_{2}=\pi^{2} \lambda_{2}^{2}\left(\frac{1}{\rho^{2}}+\frac{1}{L^{2}}\right)
$$

For any $\varphi \in L^{2}\left(\Delta_{\rho}\right)$, let

$$
F(y)=\int_{\Delta_{\rho}} w(x, y) \varphi(x) d x
$$

By (3.1.52) and by the interpolation inequality (A.1) we obtain, for every $k \geq 1$,

$$
\left|F^{(k)}(y)\right|^{2} \leq \frac{C_{3}}{L} C_{2}^{k}(k+1)^{2(k+1)}\|w\|_{L^{2}\left(Q_{0}\right)}^{2}\|\varphi\|_{L^{2}\left(\Delta_{\rho}\right)}^{2}, \quad \text { for every } y \in(-L, L),
$$

where $C_{3}$ depends on $\lambda_{2}$ and $L \rho^{-1}$ only. Therefore, for every $k \geq 1$,

$$
\int_{\Delta_{\rho}}\left|\partial_{y}^{k} w(x, y)\right|^{2} d x \leq \frac{C_{3}}{L} C_{2}^{k}(k+1)^{2(k+1)}\|w\|_{L^{2}\left(Q_{0}\right)}^{2}, \quad \text { for every } y \in(-L, L) .
$$

Let us fix $k \geq 1$ and $y \in(-L, L)$, and let us denote

$$
\begin{gathered}
g(\cdot)=b(\cdot) \partial_{y}^{k+2} w(\cdot, y), \quad \text { in } \Delta_{\rho}, \\
U(\cdot)=\partial_{y}^{k} w(\cdot, y), \quad \text { in } \Delta_{\rho} .
\end{gathered}
$$

We have that $U$ satisfies the equation

$$
\operatorname{div}(\kappa \nabla U)=-g, \quad \text { in } \Delta_{\rho}
$$

From Caccioppoli's inequality we have

$$
\|\nabla U\|_{L^{2}\left(\Delta_{\frac{3}{4} \rho}\right)}^{2} \leq C\left(\rho^{2}\|g\|_{L^{2}\left(\Delta_{\rho}\right)}^{2}+\frac{1}{\rho^{2}}\|U\|_{L^{2}\left(\Delta_{\rho}\right)}^{2}\right)
$$


where $C$ depends on $\lambda$ only. Choosing as test functions $V=\left(\eta^{2} U_{x_{i}}\right)_{x_{i}}, i=1, \ldots, n$, where $\eta$ is a cut-off function, we obtain, by standard $H^{2}$-estimates (see [GT], ch. $8])$ and by (3.1.59),

$$
\left\|D^{2} U\right\|_{L^{2}\left(\Delta_{\frac{\rho}{2}}\right)}^{2} \leq C\left(\frac{1}{\rho^{2}}+\frac{\Lambda^{2}}{R_{0}^{2}}\right)\left(\rho^{2}\|g\|_{L^{2}\left(\Delta_{\rho}\right)}^{2}+\frac{1}{\rho^{2}}\|U\|_{L^{2}\left(\Delta_{\rho}\right)}^{2}\right),
$$

where $C$ depends on $\lambda$ only. By (3.1.56), (3.1.57), (3.1.59) and (3.1.60) we have, for every $k \geq 1$,

$$
\begin{gathered}
\int_{\Delta_{\frac{3}{4} \rho}}\left|\nabla \partial_{y}^{k} w(x, y)\right|^{2} d x \leq \frac{C_{4}}{L \rho^{2}} C_{2}^{k}(k+3)^{2(k+3)}\|w\|_{L^{2}\left(Q_{0}\right)}^{2}, \\
\int_{\Delta_{\frac{\rho}{2}}}\left|D^{2} \partial_{y}^{k} w(x, y)\right|^{2} d x \leq \frac{C_{4}}{L \rho^{2}}\left(\frac{1}{\rho^{2}}+\frac{\Lambda^{2}}{R_{0}^{2}}\right) C_{2}^{k}(k+3)^{2(k+3)}\|w\|_{L^{2}\left(Q_{0}\right)}^{2},
\end{gathered}
$$

where the constant $C_{4}$ in (3.1.61) and (3.1.62) depends on $\lambda_{2}$ and $L \rho^{-1}$ only. Finally, (3.1.55), (3.1.61) and (3.1.62) yield the convergence in $H^{2}\left(\Delta_{\frac{3}{4} \rho}\right)$ of the power series (3.1.51) in the disk $D_{\rho_{1}}$, where $\rho_{1}$ is given by (3.1.47).

Let us denote, for $x \in \Delta_{\frac{3}{4} \rho}$,

$$
\begin{gathered}
W(x, z)=\sum_{0}^{\infty} \partial_{y}^{k} w(x, 0) \frac{z^{k}}{k !}, \quad \text { for } z \in D_{\rho_{1}}, \\
v(x, \xi)=W(x, i \xi), \quad \text { for }|\xi|<\rho_{1} .
\end{gathered}
$$

Step 2. For every $\xi \in\left(-\rho_{2}, \rho_{2}\right)$ we have

$$
\int_{\Delta_{\rho(\xi)}}\left(b(x) v_{\xi}^{2}(x, \xi)+\kappa \nabla v(x, \xi) \cdot \nabla v(x, \xi)\right) d x \leq \int_{\Delta_{\frac{\rho}{2}}} \kappa \nabla f(x) \cdot \nabla f(x) d x,
$$

where

$$
\rho(\xi)=\frac{\rho}{2}-\sqrt{\lambda} \lambda_{1}|\xi| .
$$

Proof of Step 2. First, let us observe that $v$ is the solution to the following Cauchy problem for a hyperbolic equation:

$$
\begin{gathered}
b(x) v_{\xi \xi}(x, \xi)-\operatorname{div}(\kappa(x) \nabla v(x, \xi))=0, \quad \text { in } \Delta_{\frac{\rho}{2}} \times\left(-\rho_{1}, \rho_{1}\right), \\
v(x, 0)=f(x), \quad \text { in } \Delta_{\frac{\rho}{2}}, \\
v_{\xi}(x, 0)=0, \quad \text { in } \Delta_{\frac{\rho}{2}} .
\end{gathered}
$$

We shall derive estimate (3.1.63) from an energy estimate for equation (3.1.65a). To this aim, let us denote

$$
E(\xi)=\frac{1}{2} \int_{\Delta_{\rho(\xi)}}\left(b(x) v_{\xi}^{2}(x, \xi)+\kappa(x) \nabla v(x, \xi) \cdot \nabla v(x, \xi)\right) d x .
$$

Since $\xi \rightarrow v(\cdot, \xi)$ is an analytic function from $\left(-\rho_{1}, \rho_{1}\right)$ to $H^{2}\left(\Delta_{\frac{\rho}{2}}\right)$, we have that $\partial_{\xi}^{k} v(\cdot, \xi) \in H^{3 / 2}\left(\partial \Delta_{\rho(\xi)}\right)$ for every $\xi \in\left(-\rho_{2}, \rho_{2}\right)$ and for every $k \geq 1$, where $\rho_{2}$ is 
given by (3.1.48). Therefore, for every $\xi \in\left(-\rho_{2}, \rho_{2}\right)$, we may write the following equalities:

$$
\begin{aligned}
E(\xi) & =\frac{1}{2} \int_{0}^{\rho(\xi)} d \eta \int_{\partial \Delta_{\eta}}\left(b(x) v_{\xi}^{2}(x, \xi)+\kappa(x) \nabla v(x, \xi) \cdot \nabla v(x, \xi)\right) d s \\
E^{\prime}(\xi) & =\int_{0}^{\rho(\xi)} d \eta \int_{\partial \Delta_{\eta}}\left(b(x) v_{\xi}(x, \xi) v_{\xi \xi}(x, \xi)+\kappa(x) \nabla v(x, \xi) \cdot \nabla v_{\xi}(x, \xi)\right) d s \\
& -\frac{\sqrt{\lambda} \lambda_{1}}{2} \int_{\partial \Delta_{\rho(\xi)}}\left(b(x) v_{\xi}^{2}(x, \xi)+\kappa(x) \nabla v(x, \xi) \cdot \nabla v(x, \xi)\right) d s \\
& =\int_{\partial \Delta_{\rho(\xi)}} \kappa(x) \nabla v(x, \xi) \cdot \nu v_{\xi}(x, \xi) d s \\
- & \frac{\sqrt{\lambda} \lambda_{1}}{2} \int_{\partial \Delta_{\rho(\xi)}}\left(b(x) v_{\xi}^{2}(x, \xi)+\kappa(x) \nabla v(x, \xi) \cdot \nabla v(x, \xi)\right) d s
\end{aligned}
$$

where $\nu$ denotes the outer unit normal to $\Delta_{\rho(\xi)}$. We have

$$
\begin{gathered}
\left|\kappa(x) \nabla v(x, \xi) \cdot \nu v_{\xi}(x, \xi)\right| \leq(\kappa(x) \nabla v(x, \xi) \cdot \nabla v(x, \xi))^{1 / 2}(\kappa(x) \nu \cdot \nu)^{1 / 2}\left|v_{\xi}\right| \\
\leq \frac{\sqrt{\lambda}}{2}\left(v_{\xi}^{2}(x, \xi)+\kappa(x) \nabla v(x, \xi) \cdot \nabla v(x, \xi)\right) .
\end{gathered}
$$

Therefore

$$
\begin{gathered}
E^{\prime}(\xi) \leq \frac{\sqrt{\lambda}}{2} \int_{\partial \Delta_{\rho(\xi)}}\left(v_{\xi}^{2}(x, \xi)+\kappa(x) \nabla v(x, \xi) \cdot \nabla v(x, \xi)\right) d s \\
-\frac{\sqrt{\lambda} \lambda_{1}}{2} \int_{\partial \Delta_{\rho(\xi)}}\left(\lambda_{1}^{-1} v_{\xi}^{2}(x, \xi)+\kappa(x) \nabla v(x, \xi) \cdot \nabla v(x, \xi)\right) d s \\
=\frac{\sqrt{\lambda}}{2}\left(1-\lambda_{1}\right) \int_{\partial \Delta_{\rho(\xi)}}(\kappa(x) \nabla v(x, \xi) \cdot \nabla v(x, \xi) d s \leq 0 .
\end{gathered}
$$

Hence $E(\cdot)$ is decreasing, so that $E(\xi) \leq E(0)$ and (3.1.63) follows.

Proof of Proposition 3.1.4 (Conclusion). For every $z \in D_{\rho_{1}}$ let us set

$$
G(z)=\int_{\Delta_{\rho_{3}}}\left(b(x) W_{z}^{2}(x, z)+\kappa(x) \nabla W(x, z) \cdot \nabla W(x, z)\right) d x,
$$

and let

$$
\epsilon^{2}=\int_{\Delta_{\frac{\rho}{2}}} \kappa(x) \nabla f(x) \cdot \nabla f(x) d x .
$$

Let $\rho_{1}^{\prime} \in\left(0, \rho_{1}\right)$. By (3.1.55) and (3.1.61) we obtain

$$
|G(z)| \leq \frac{C}{L \rho^{2}\left(1-\rho_{1}^{\prime} \rho_{1}^{-1}\right)^{8}}\|w\|_{L^{2}\left(Q_{0}\right)}^{2}, \quad \text { for every } z \in D_{\rho_{1}^{\prime}},
$$

where $C$ depends on $\lambda_{2}$ and $L \rho^{-1}$ only. On the other side (3.1.63) gives

$$
|G(i \xi)| \leq \epsilon^{2}, \quad \text { for every } \xi \in\left(-\rho_{2}, \rho_{2}\right) \text {. }
$$


From (3.1.66), (3.1.67) and the analytic continuation estimate (see [Is1]) we obtain

$$
\begin{gathered}
\int_{\Delta_{\rho_{3}}}\left(b(x) w_{y}^{2}(x, y)+\kappa(x) \nabla w(x, y) \cdot \nabla w(x, y)\right) d x=G(y) \\
\leq \frac{1}{\left(1-\rho_{1}^{\prime} \rho_{1}^{-1}\right)^{8}}\left(\frac{C}{L \rho^{2}}\|w\|_{L^{2}\left(Q_{0}\right)}^{2}+\int_{\Delta_{\frac{\rho}{2}}} \kappa(x) \nabla f(x) \cdot \nabla f(x) d x\right)^{1-\omega(y, 0)} \\
\times\left(\int_{\Delta_{\frac{\rho}{2}}} \kappa(x) \nabla f(x) \cdot \nabla f(x) d x\right)^{\omega(y, 0)},
\end{gathered}
$$

where $\omega(y, \xi)$ is the harmonic measure of $\left\{i \xi\right.$ s.t. $\left.\xi \in\left[-\frac{\rho_{2}}{2}, \frac{\rho_{2}}{2}\right]\right\}$ with respect to $\left\{y+i \xi \in \mathbb{C}\right.$ s.t. $\left.y^{2}+\xi^{2}=\left(\rho_{1}^{\prime}\right)^{2}\right\}$ and $C$ depends on $\lambda_{2}$ and $L \rho^{-1}$ only. Now, let us choose $\rho_{1}^{\prime}=\frac{3}{4} \rho_{1}$, so that $\frac{\rho_{2}}{2}<\rho_{1}^{\prime}<\rho_{1}$. We have that $\omega(y, 0) \geq \beta>0$ for every $y \in\left(-\frac{3}{8} \rho_{1}, \frac{3}{8} \rho_{1}\right)$, where $\beta$ depends on $\lambda$ and $\Lambda$ only. Therefore estimate (3.1.50) follows by (3.1.68).

Lemma 3.1.5. Let $w$ be the solution to the Cauchy problem

$$
\begin{gathered}
\operatorname{div}(\kappa(x) \nabla w(x, y))+b(x) w_{y y}(x, y)=0, \quad \text { in } \Delta_{R} \times(-\sqrt{2} \delta,+\sqrt{2} \delta), \\
w(x, 0)=f(x), \quad \text { in } \Delta_{R}, \\
w_{y}(x, 0)=0, \quad \text { in } \Delta_{R},
\end{gathered}
$$

where $\kappa$ satisfies (3.1.1), $b$ satisfies (3.1.2) and $\delta$ is given by (3.1.32). For every $r \leq \theta_{1} R$ we have

$$
\int_{B_{r}} w^{2} d x d y \leq C r^{\bar{\beta}}\left(\int_{B_{\tilde{\rho}}} w^{2} d x d y\right)^{1-\bar{\beta}}\left(\int_{\Delta_{\rho / 2}} f^{2} d x\right)^{\bar{\beta}},
$$

where $\theta_{1}, 0<\theta_{1}<1, \bar{\beta}, 0<\bar{\beta}<1$ and $C \geq 1$ depend on $\lambda_{2}$ only, $\rho=\frac{8}{3} \sqrt{2} e \pi \lambda_{2} r$ and $\tilde{\rho}=2 \sqrt{2} \rho$.

Proof of Lemma 3.1.5. Let us denote

$$
\theta_{1}=\frac{3}{\sqrt{2}\left(8 e \pi \lambda_{2}\right)^{2}},
$$

and let $r$ be such that $0<r \leq \theta_{1} R$. Let us choose

$$
\rho=L=\frac{8 \sqrt{2}}{3} e \pi \lambda_{2} r
$$

in estimate (3.1.50). This choice gives $r=\frac{3}{8} \rho_{1}$, where $\rho_{1}$ is defined by (3.1.47). Let us denote

$$
\tilde{\rho}=2 \sqrt{2} \rho,
$$

and let us notice that $\tilde{\rho}<\sqrt{2} \delta$. We have

$$
B_{r} \subset \Delta_{\rho / 4} \times\left(-\frac{3}{8} \rho_{1}, \frac{3}{8} \rho_{1}\right) \subset \Delta_{2 \rho} \times(-2 L, 2 L) \subset B_{\tilde{\rho}} \subset \Delta_{R} \times(-\sqrt{2} \delta, \sqrt{2} \delta) .
$$


Integrating both the sides of inequality (3.1.50), we obtain, by the inclusions (3.1.71),

$$
\int_{B_{r}^{+}}|\nabla w|^{2} d x d y \leq C r\left(\frac{1}{r^{3}}\|w\|_{L^{2}\left(B_{\tilde{\rho}}^{+}\right)}^{2}+\|\nabla f\|_{L^{2}\left(\Delta_{\rho / 2}\right)}^{2}\right)^{1-\beta}\|\nabla f\|_{L^{2}\left(\Delta_{\rho / 2}\right)}^{2 \beta},
$$

where $C$ only depends on $\lambda_{2}$. By the standard elliptic estimate (see, for instance, GT] )

$$
\rho^{1+\alpha}|\nabla w|_{\alpha, B_{\frac{3}{4} \rho}^{+} \rho} \leq \frac{C}{\rho^{\frac{n+1}{2}}}\|w\|_{L^{2}\left(B_{\rho}^{+}\right)}, \quad \text { for every } \alpha \in(0,1),
$$

where $C$ depends on $\lambda_{2}, \Lambda_{2}$ and $\alpha$ only, and by (A.3) we obtain

$$
\rho^{2} \int_{\Delta \rho / 2}|\nabla f|^{2} d x \leq C\left(\int_{\Delta \rho / 2} f^{2} d x\right)^{\frac{\alpha}{1+\alpha}}\left(\int_{\Delta \rho / 2} f^{2} d x+\frac{1}{\rho}\|w\|_{L^{2}\left(B_{\rho}^{+}\right)}^{2}\right)^{\frac{1}{1+\alpha}},
$$

where $C$ depends on $\lambda_{2}, \Lambda_{2}$ and $\alpha$ only. By (A.4) and by the Caccioppoli inequality we have

$$
\int_{\Delta \rho / 2} f^{2} d x \leq c\left(\frac{1}{\rho} \int_{B_{\frac{3}{4} \rho}^{+}} w^{2} d x d y+\rho \int_{B_{\frac{3}{4} \rho}^{+} \rho}|\nabla w|^{2} d x d y\right) \leq \frac{C}{\rho} \int_{B_{\rho}^{+}} w^{2} d x d y,
$$

where $C$ depends on $\lambda_{2}$ only. By (3.1.74) and (3.1.75), we have

$$
\rho^{2} \int_{\Delta \rho / 2}|\nabla f|^{2} d x \leq C\left(\int_{\Delta \rho / 2} f^{2} d x\right)^{\frac{\alpha}{1+\alpha}}\left(\frac{1}{\rho}\|w\|_{L^{2}\left(B_{\rho}^{+}\right)}^{2}\right)^{\frac{1}{1+\alpha}}
$$

where $C$ depends on $\lambda_{2}, \Lambda_{2}$ and $\alpha$ only. By (3.1.75) and (3.1.76), we have

$$
\rho^{2} \int_{\Delta \rho / 2}|\nabla f|^{2} d x \leq \frac{C}{\rho}\|w\|_{L^{2}\left(B_{\rho}^{+}\right)}^{2},
$$

where $C$ depends on $\lambda_{2}, \Lambda_{2}$ and $\alpha$ only. By (3.1.72), (3.1.76) and (3.1.77), we obtain

$$
\int_{B_{r}^{+}}|\nabla w|^{2} d x d y \leq C r\left(\frac{1}{r^{2}} \int_{\Delta \rho / 2} f^{2} d x\right)^{\bar{\beta}}\left(\frac{1}{r^{3}}\|w\|_{L^{2}\left(B_{\bar{\rho}}^{+}\right)}^{2}\right)^{1-\bar{\beta}},
$$

where $C$ depends on $\lambda_{2}, \Lambda_{2}$ and $\alpha$ only and $\bar{\beta}=\frac{\beta \alpha}{1+\alpha}$. By (A.5), (3.1.78) and (3.1.75) we have

$$
\begin{gathered}
\int_{B_{r}^{+}} w^{2} d x d y \leq c\left(r \int_{\Delta_{r}} f^{2} d x+r^{2} \int_{B_{r}^{+}}|\nabla w|^{2} d x d y\right) \\
\leq C r\left(\int_{\Delta_{\rho / 2}} f^{2} d x\right)^{\bar{\beta}}\left(\frac{1}{r}\|w\|_{L^{2}\left(B_{\bar{\rho}}^{+}\right)}^{2}\right)^{1-\bar{\beta}},
\end{gathered}
$$

where $C$ depends on $\lambda_{2}, \Lambda_{2}$ and $\alpha$ only. Choosing $\alpha=\frac{1}{2}$, we obtain (3.1.70). 
Now, let us recall the three spheres inequality for elliptic equations. To this aim, let as assume that $\sigma(x)$ is a given function from the ball in $\mathbb{R}^{m} B_{\bar{R}}$ with values $m \times m$ symmetric matrices satisfying the following conditions:

$$
\lambda^{-1}|\xi|^{2} \leq \sigma(x) \xi \cdot \xi \leq \lambda|\xi|^{2}, \quad \text { for every } x \in B_{\bar{R}} \text { and } \xi \in \mathbb{R}^{m}, \quad \text { (ellipticity) }
$$

$$
\left|\sigma\left(x^{\prime}\right)-\sigma\left(x^{\prime \prime}\right)\right| \leq \frac{\Lambda}{R_{0}}\left|x^{\prime}-x^{\prime \prime}\right|, \quad \text { for every } x^{\prime}, x^{\prime \prime} \in B_{\bar{R}} . \quad \text { (Lipschitz continuity) }
$$

The proof of the following Lemma 3.1.6 follows from $[\mathrm{K}]$ with some slight changes.

Lemma 3.1.6 (Kukavica). Let $w \in H^{1}\left(B_{\bar{R}}\right)$ be a solution to

$$
\operatorname{div}(\sigma(x) \nabla w(x))=0, \quad \text { in } B_{\bar{R}}
$$

where $\sigma$ satisfies (3.1.80). For every $0<r_{1}<r_{2}<\frac{r_{3}}{2 \lambda} \leq \theta_{2} \bar{R}$ we have

$$
\int_{B_{r_{2}}(0)} w^{2}(x) d x
$$

$$
\leq C\left(\frac{r_{3}}{r_{2}}\right)^{C}\left(\int_{B_{r_{1}}(0)} w^{2}(x) d x\right)^{\gamma\left(r_{1}, r_{2}, r_{3}\right)}\left(\int_{B_{r_{3}}(0)} w^{2}(x) d x\right)^{1-\gamma\left(r_{1}, r_{2}, r_{3}\right)}
$$

where

$$
\gamma\left(r_{1}, r_{2}, r_{3}\right)=\frac{\log \left(\frac{1}{2}+\frac{r_{3}}{2 \lambda r_{2}}\right)}{\log \left(\frac{1}{2}+\frac{r_{3}}{2 \lambda r_{2}}\right)+C \log \frac{2 \lambda r_{2}}{r_{1}}},
$$

and $\theta_{2}, 0<\theta_{2}<1$, depends on $\Lambda$ only and $C>0$ depends on $\lambda$ and $\Lambda$ only.

Proof of Theorem 3.1.1. For fixed $t_{0} \in(0, T)$, let us consider the function $w$ introduced above, which satisfies (3.1.45). Let $r_{1}, r_{2}, r_{3}$ be such that

$$
0<r_{1}<r_{2}<\left(4 \max \left\{\sqrt{2}, \lambda_{2}\right\}\right)^{-1} r_{3}<r_{3} \leq \bar{\theta} R,
$$

with $\bar{\theta}=\min \left\{\theta_{1}, \frac{\theta_{2} \delta}{\sqrt{2} \lambda_{2} R}\right\}$, where $\theta_{1}, \theta_{2}$ have been introduced in Lemma 3.1.5 and Lemma 3.1.6 respectively and $\delta$ is given by (3.1.32). Let $r_{2}^{\prime}=(1-\mu) r_{2}+\mu r_{3}$, where $\mu=\frac{1}{4 \lambda_{2}-1}$, and let $a=\frac{3}{4 \sqrt{2} e \pi \lambda_{2}}$. We have that $0<a r_{1}<r_{1}<r_{2}<r_{2}^{\prime}<\frac{r_{3}}{2 \lambda_{2}}$. By applying Lemma 3.1.6 to the triplet of radii $a r_{1}, r_{2}^{\prime}, r_{3}$, we obtain

$$
\int_{B_{r_{2}^{\prime}}} w^{2} d x d y \leq C\left(\frac{r_{3}}{r_{2}}\right)^{C}\left(\int_{B_{a r_{1}}} w^{2} d x d y\right)^{\gamma^{\prime}}\left(\int_{B_{r_{3}}} w^{2} d x d y\right)^{1-\gamma^{\prime}}
$$

where $\gamma^{\prime}=\gamma\left(a r_{1}, r_{2}^{\prime}, r_{3}\right)$, with $\gamma$ defined by (3.1.83), and where $C$ depends on $\lambda_{2}$ and $\Lambda_{2}$ only. Let $\bar{r}_{2}=\frac{1}{2}\left(r_{2}+r_{2}^{\prime}\right)$. By the Caccioppoli inequality, by (A.4) and by the choice of $\mu$, it follows that

$$
\begin{aligned}
& \int_{B_{r_{2}}} w^{2} d x d y=\frac{1}{2} \int_{B_{r_{2}^{\prime}}} w^{2} d x d y+\frac{1}{2} \int_{B_{r_{2}^{\prime}}} w^{2} d x d y \\
& \geq \frac{1}{2} \int_{B_{\bar{r}_{2}}} w^{2} d x d y+\frac{c}{\lambda_{2}^{4}}\left(r_{2}^{\prime}-\bar{r}_{2}\right)^{2} \int_{B_{\bar{r}_{2}}}|\nabla w|^{2} d x d y
\end{aligned}
$$




$$
\begin{aligned}
& \geq C\left(r_{3}-r_{2}\right)\left(\frac{\bar{r}_{2}}{\bar{r}_{2}^{2}-r_{2}^{2}} \int_{B_{\bar{r}_{2}}} w^{2} d x d y+\bar{r}_{2} \int_{B_{\bar{r}_{2}}}|\nabla w|^{2} d x d y\right) \\
& \quad \geq C\left(r_{3}-r_{2}\right) \int_{\Delta_{r_{2}}} w^{2}(x, 0) d x=C\left(r_{3}-r_{2}\right) \int_{\Delta_{r_{2}}} u^{2}\left(x, t_{0}\right) d x
\end{aligned}
$$

where $C$ depends on $\lambda_{2}$ only. On the other hand, by applying Lemma 3.1.5 we can estimate the integral $\int_{B_{a r_{1}}} w^{2} d x d y$ appearing in (3.1.84), obtaining

$$
\int_{B_{r_{2}^{\prime}}} w^{2} d x d y \leq C\left(\frac{r_{3}}{r_{2}}\right)^{C} r_{1}^{\bar{\beta} \gamma^{\prime}}\left(\int_{\Delta_{r_{1}}} u^{2}\left(x, t_{0}\right) d x\right)^{\bar{\beta} \gamma^{\prime}}\left(\int_{B_{r_{3}}} w^{2} d x d y\right)^{1-\bar{\beta} \gamma^{\prime}}
$$

where $C$ depends on $\lambda_{2}$ and $\Lambda_{2}$ only. Now, let us estimate $\int_{B_{r_{3}}} w^{2} d x d y$. By the definition of $w$, and recalling that $r_{3} \leq \frac{\delta}{2}$, we have

$$
|w(x, y)|=\frac{1}{2 \pi} \int_{-\infty}^{+\infty}|\hat{u}(x, \mu)| e^{\sqrt{|\mu| / 2} r_{3}} d \mu \leq C(\delta)\left(\int_{-\infty}^{+\infty}|\hat{u}(x, \mu)|^{2} e^{\delta \sqrt{|\mu|}} d \mu\right)^{1 / 2}
$$

where

$$
C(\delta)=\frac{1}{2 \pi}\left(\int_{-\infty}^{+\infty} e^{\left(\frac{1}{\sqrt{2}}-1\right) \delta \sqrt{|\mu|}} d \mu\right)^{1 / 2}=\left(1-\frac{1}{\sqrt{2}}\right)^{-1} \frac{1}{\pi \delta} .
$$

By (3.1.31) and (3.1.87) we have

$$
\begin{gathered}
\int_{\Delta_{r_{3}}} w^{2}(x, y) d x \leq C^{2}(\delta) \int_{-\infty}^{+\infty} \int_{\Delta_{r_{3}}}|\hat{u}(x, \mu)|^{2} e^{\delta \sqrt{|\mu|}} d x d \mu \\
\leq c C^{2}(\delta) C_{1}^{2} \lambda_{2}^{2} H^{2}\left(T+\frac{1}{a_{R}}\right)^{2} \int_{-\infty}^{+\infty} e^{-\delta \sqrt{|\mu|}} d \mu .
\end{gathered}
$$

Since $\int_{-\infty}^{+\infty} e^{-\delta \sqrt{|\mu|}} d \mu=\frac{4}{\delta^{2}}$, from the definition of $a_{R}$ and $\delta$ and from (3.1.88) we obtain

$$
\int_{B_{r_{3}}} w^{2} d x d y \leq \int_{0}^{r_{3}} \int_{\Delta_{r_{3}}} w^{2} d x d y \leq C C_{1}^{2} r_{3} H^{2}\left(\frac{T^{2}}{R^{4}}+1\right),
$$

where $C$ depends on $\lambda_{2}$ only. From (3.1.85), (3.1.86) and (3.1.89), choosing $t_{0} \in$ $\left(0, \frac{T}{2}\right)$ in order to control the constant $C_{1}$ given by $(3.1 .13)$, we obtain $(3.1 .7 a)$. By integrating $(3.1 .7 \mathrm{a})$ over $\left(0, \frac{T}{2}\right)$ and using the Hölder inequality we obtain $(3.1 .7 \mathrm{~b})$.

Theorem 3.1.1'. (Three Spheres Inequalities and Three Cylinders Inequalities in the Interior). Let $u \in H^{2,1}\left(\Delta_{2 R} \times(0, T)\right)$ be a solution to $(3.1 .5 \mathrm{a})$ and let the assumptions (3.1.1) - (3.1.2) be satisfied. For every $r_{1}, r_{2}, r_{3}$ such that $0<r_{1}<$ 
$r_{2}<\left(4 \max \left\{\sqrt{2}, \lambda_{2}\right\}\right)^{-1} r_{3}<r_{3} \leq \hat{\theta}_{1} R$, we have

$$
\int_{\Delta_{r_{2}}} u^{2}\left(x, t_{0}\right) d x
$$

$$
\leq \tilde{C}_{1} \frac{r_{3}}{r_{3}-r_{2}}\left(\frac{r_{3}}{r_{2}}\right)^{C}\left(\left(1+\frac{T^{2}}{R^{4}}\right) H^{2}\right)^{1-\bar{\beta} \gamma^{\prime}}\left(\int_{\Delta_{r_{1}}} u^{2}\left(x, t_{0}\right) d x\right)^{\bar{\beta} \gamma^{\prime}}, \forall t_{0} \in(0, T),
$$

where $H$ and $\gamma^{\prime}$ are defined by (3.1.6) and (3.1.8) respectively, $\bar{\beta}, 0<\bar{\beta}<1$, depends on $\lambda_{2}$ only, $C$ depends on $\lambda_{2}$ and $\Lambda_{2}$ only, $\hat{\theta}_{1}, 0<\hat{\theta}_{1}<1$, depends on $\lambda_{2}, \Lambda_{2}$ and $\frac{t_{0}}{R^{2}}$ only, $\hat{\theta}_{1}=O\left(t_{0}\right)$ as $t_{0} \rightarrow 0$, and $\tilde{C}_{1}$ depends on $\lambda_{2}, \Lambda_{2}, \frac{R_{0}^{2}}{T}$ and $\frac{T}{T-t_{0}}$ only. Let $\sigma \in\left(0, \frac{1}{2}\right)$. For every $r_{1}, r_{2}, r_{3}$ such that $0<r_{1}<r_{2}<\left(4 \max \left\{\sqrt{2}, \lambda_{2}\right\}\right)^{-1} r_{3}<$ $r_{3} \leq \hat{\theta}_{2} R$, we have

$$
\int_{\sigma T}^{(1-\sigma) T} \int_{\Delta_{r_{2}}} u^{2}(x, t) d x d t
$$

$$
\leq \tilde{C}_{2} \frac{r_{3}}{r_{3}-r_{2}}\left(\frac{r_{3}}{r_{2}}\right)^{C}\left(T\left(1+\frac{T^{2}}{R^{4}}\right) H^{2}\right)^{1-\bar{\beta} \gamma^{\prime}}\left(\int_{\sigma T}^{(1-\sigma) T} \int_{\Delta_{r_{1}}} u^{2}(x, t) d x d t\right)^{\bar{\beta} \gamma^{\prime}},
$$

where $H, \gamma^{\prime}, \bar{\beta}$ and $C$ are as above, $\hat{\theta}_{2}, 0<\hat{\theta}_{2}<1$, depends on $\lambda_{2}, \Lambda_{2}, \sigma$ and $\frac{T}{R^{2}}$ only, and $\tilde{C}_{2}$ depends on $\lambda_{2}, \Lambda_{2}, \frac{R_{0}^{2}}{T}$ and $\sigma$ only.

Proof. Let us fix $t_{0} \in(0, T)$ and, let us denote by $u_{1}, u_{2}$ the weak solutions to problem (3.1.9) and to the following problem:

$$
\begin{gathered}
b(x) u_{2, t}(x, t)-\operatorname{div}\left(\kappa(x) \nabla u_{2}(x, t)\right)=0, \quad \text { in } \Delta_{2 R} \times(0,+\infty), \\
u_{2}=0, \quad \text { on } \partial \Delta_{2 R} \times[0,+\infty), \\
u_{2}=u, \quad \text { on } \Delta_{2 R} \times\{0\},
\end{gathered}
$$

respectively. We have that $u=u_{1}+u_{2}$ in $\Delta_{2 R} \times\left[0, t_{0}\right]$ and, in particular,

$$
u\left(\cdot, t_{0}\right)=u_{1}\left(\cdot, t_{0}\right)+u_{2}\left(\cdot, t_{0}\right), \quad \text { in } \Delta_{2 R} .
$$

By some slight changes in the proofs of Lemma 3.1.2 and Lemma 3.1.3, we have that $(3.1 .12)-(3.1 .13)$ and $(3.1 .31)-(3.1 .32)$ continue to hold if we replace $\tilde{u}$ by $u_{1}$ and $\hat{u}(x, \mu)$ by

$$
\hat{u}_{1}(x, \mu)=\int_{-\infty}^{+\infty} e^{-\mu i t} u_{1}(x, t) d t=\int_{0}^{+\infty} e^{-\mu i t} u_{1}(x, t) d t, \quad \text { for every } \mu \in \mathbb{R} .
$$

Moreover, the function $w_{1}(x, y)=\frac{1}{2 \pi} \int_{-\infty}^{+\infty} e^{i t_{0} \mu} \hat{u}_{1}(x, \mu) \cosh \sqrt{-i \mu} y d \mu$, defined for $x \in \Delta_{R},|y|<\sqrt{2} \delta$, with $\delta$ given by (3.1.32), is a solution to the elliptic equation (3.1.45a) and satisfies the following conditions:

$$
\begin{gathered}
w_{1}(x, 0)=u_{1}\left(x, t_{0}\right), \quad \text { in } \Delta_{R}, \\
w_{1, y}(x, 0)=0, \quad \text { in } \Delta_{R} .
\end{gathered}
$$

Now, let us define a continuation of $u_{2}\left(\cdot, t_{0}\right)$ to a solution of the elliptic equation (3.1.45a). Let $\mu_{k}, \varphi_{k}$ be the eigenvalues and eigenfunctions associated to problem 
(3.1.20), respectively (as in the proof of Lemma 3.1.2), and let $\alpha_{k}$ be the Fourier coefficients

$$
\alpha_{k}=\int_{\Delta_{2 R}} u(x, 0) b(x) \varphi_{k}(x) d x
$$

We have

$$
u_{2}(x, t)=\sum_{k=1}^{\infty} \alpha_{k} e^{\mu_{k} t} \varphi_{k}(x), \quad \text { in } \Delta_{2 R} \times(0,+\infty) .
$$

Let us define

$$
w_{2}(x, y)=\sum_{k=1}^{\infty} \alpha_{k} e^{\mu_{k} t_{0}} \varphi_{k}(x) \cosh \sqrt{\left|\mu_{k}\right|} y, \quad \text { in } \Delta_{2 R} \times\left[-t_{0} \sqrt{a_{R}}, t_{0} \sqrt{a_{R}}\right] .
$$

We have that $w_{2} \in H_{l o c}^{1}\left(\Delta_{2 R} \times\left[-t_{0} \sqrt{a_{R}}, t_{0} \sqrt{a_{R}}\right]\right)$ satisfies

$$
\begin{gathered}
\operatorname{div}\left(\kappa(x) \nabla w_{2}(x, y)\right)+b(x) w_{2, y y}(x, y)=0, \quad \text { in } \Delta_{2 R} \times\left[-t_{0} \sqrt{a_{R}}, t_{0} \sqrt{a_{R}}\right], \\
w_{2}(x, 0)=u_{2}\left(x, t_{0}\right), \quad \text { in } \Delta_{2 R}, \\
w_{2, y}(x, 0)=0, \quad \text { in } \Delta_{2 R},
\end{gathered}
$$

and moreover $w_{2}$ is even with respect to the variable $y$.

Setting $\bar{\delta}=\min \left\{\sqrt{2} \delta, t_{0} \sqrt{a_{R}}\right\}=C R$, with $C$ only depending on $\lambda, \lambda_{1}, \frac{t_{0}}{R^{2}}$, and defining

$$
w=w_{1}+w_{2} \text { in } \Delta_{R} \times(-\bar{\delta},+\bar{\delta}),
$$

we obtain that $w \in H_{l o c}^{1}\left(\Delta_{R} \times(-\bar{\delta}, \bar{\delta})\right)$ satisfies the following elliptic equation

$$
\operatorname{div}(\kappa(x) \nabla w(x, y))+b(x) w_{y y}(x, y)=0, \quad \text { in } \Delta_{R} \times(-\bar{\delta},+\bar{\delta}),
$$

and the conditions

$$
\begin{gathered}
w(x, 0)=u\left(x, t_{0}\right), \quad \text { in } \Delta_{R}, \\
w_{y}(x, 0)=0, \quad \text { in } \Delta_{R} .
\end{gathered}
$$

Moreover, $w$ is even with respect to the variable $y$. Let us prove that

$$
\int_{B_{r}^{+}} w_{2}^{2} d x d y \leq \lambda_{1}^{2} r H^{2}
$$

for $r \leq \min \left\{\sqrt{a_{R}} t_{0}, 2 R\right\}$.

We have

$$
\begin{gathered}
\int_{B_{r}^{+}} w_{2}^{2} d x d y \leq \lambda_{1} \int_{0}^{r}\left(\int_{\Delta_{2 R}} w_{2}^{2}(x, y) b(x) d x\right) d y \\
=\lambda_{1} \int_{0}^{r} \sum_{k=1}^{\infty} \alpha_{k}^{2} e^{2 \mu_{k} t_{0}} \cosh ^{2} \sqrt{\left|\mu_{k}\right|} y d y \leq \lambda_{1} r \sum_{k=1}^{\infty} \alpha_{k}^{2} e^{-2 \sqrt{\left|\mu_{k}\right|}\left(\sqrt{\left|\mu_{k}\right|} t_{0}-r\right)} \\
\leq \lambda_{1} r \sum_{k=1}^{\infty} \alpha_{k}^{2}=\lambda_{1} r \int_{\Delta_{2 R}} u^{2}(x, 0) b(x) d x \leq \lambda_{1}^{2} r H^{2} .
\end{gathered}
$$

Now, let $r_{1}, r_{2}, r_{3}$ be such that $0<r_{1}<r_{2}<\left(4 \max \left\{\sqrt{2}, \lambda_{2}\right\}\right)^{-1} r_{3}<r_{3} \leq \hat{\theta}_{1} R$, where $\hat{\theta}_{1}=\min \left\{\bar{\theta}, \frac{1}{\lambda_{2} \sqrt{c_{P}}} \frac{t_{0}}{R^{2}}\right\}$, where $\bar{\theta}$ has been introduced in Theorem 3.1.1. By 
(3.1.92) and by noticing that estimate (3.1.89) in the proof of Theorem 3.1.1 holds also for $w_{1}$, with the same values of the constants, we obtain

$$
\int_{B_{r_{3}}} w^{2} d x d y \leq C C_{1}^{2} r_{3} H^{2}\left(\frac{T^{2}}{R^{4}}+1\right),
$$

where $C$ depends on $\lambda_{2}$ only and $C_{1}$ is defined by (3.1.13). From this point we can repeat the proof of Theorem 3.1.1, obtaining (3.1.90a), with the stated dependence of the constants. Now, let $\sigma \in\left(0, \frac{1}{2}\right)$ and let $r_{1}, r_{2}, r_{3}$ be such that $0<r_{1}<r_{2}<$ $\left(4 \max \left\{\sqrt{2}, \lambda_{2}\right\}\right)^{-1} r_{3}<r_{3} \leq \hat{\theta}_{2} R$, with $\hat{\theta}_{2}=\min \left\{\bar{\theta}, \frac{1}{\lambda_{2} \sqrt{c_{P}}} \frac{\sigma T}{R^{2}}\right\}$. By integrating (3.1.90a) over $(\sigma T,(1-\sigma) T)$ and using the Hölder inequality, we obtain (3.1.90b).

Corollary 3.1.7. (Strong Unique Continuation on the Characteristic Planes $t=$ $\left.t_{0}\right)$. Let $u \in H^{2,1}\left(\Delta_{2 R} \times(0, T)\right)$ be a solution to $(3.1 .5 \mathrm{a})$ and let $(3.1 .1)-(3.1 .2)$ be satisfied. Let $t_{0} \in(0, T)$. If, for every $k \in \mathbb{N}$,

$$
\int_{\Delta_{r}} u^{2}\left(x, t_{0}\right) d x=O\left(r^{k}\right), \quad \text { as } r \rightarrow 0,
$$

then

$$
u\left(\cdot, t_{0}\right) \equiv 0, \quad \text { in } \Delta_{2 R} .
$$

Proof. Let $r_{3}=\hat{\theta}_{1} R, r_{2}=\frac{1}{2}\left(4 \max \left\{\sqrt{2}, \lambda_{2}\right\}\right)^{-1} r_{3}$. Let us fix $k \in \mathbb{N}$. By (3.1.93) we have, for $r_{1}$ small enough,

$$
R^{-n} \int_{\Delta_{r_{1}}} u^{2}\left(x, t_{0}\right) d x \leq\left(\frac{r_{1}}{R}\right)^{k} .
$$

By Theorem 3.1.1' and by (3.1.95) we have

$$
R^{-n} \int_{\Delta_{r_{2}}} u^{2}\left(x, t_{0}\right) d x \leq C^{\prime}\left(R^{-n} H^{2}\right)^{1-\bar{\beta} \gamma^{\prime}}\left(\frac{r_{1}}{R}\right)^{k \bar{\beta} \gamma^{\prime}}
$$

where $\bar{\beta}$ and $\gamma^{\prime}$ are the same of Theorem $3.1 .1^{\prime}$ and $C^{\prime}$ depends on on $\lambda_{2}, \Lambda_{2}, \frac{R^{2}}{T}$ and $\frac{T}{T-t_{0}}$ only. Passing to the limit in (3.1.96) as $r_{1} \rightarrow 0$, we have

$$
R^{-n} \int_{\Delta_{r_{2}}} u^{2}\left(x, t_{0}\right) d x \leq C^{\prime}\left(R^{-n} H^{2}\right)^{1-\bar{\beta} \gamma^{\prime}} e^{-\bar{C} k},
$$

where $\bar{C}>0$ depends on $\lambda_{2}$ and $\Lambda_{2}$ only. Now, passing to the limit in (3.1.97) as $k \rightarrow \infty$, we obtain

$$
R^{-n} \int_{\Delta_{r_{2}}} u^{2}\left(x, t_{0}\right) d x=0
$$

so that $u\left(\cdot, t_{0}\right) \equiv 0$ in $\Delta_{r_{2}}$. By iteration, (3.1.94) follows.

Subsection 3.2 (Three Spheres Inequalities and Three Cylinders Inequalities at the Boundary).

Theorem 3.2.1. (Three Spheres Inequalities and Three Cylinders Inequalities at the Boundary). Let $\Omega$ be a domain in $\mathbb{R}^{n}$ such that

$$
\partial \Omega \text { is of class } C^{1,1} \text { with constants } R_{0}, E \text {. }
$$


Let $u \in H^{2,1}(\Omega \times(0, T))$ be a solution to

$$
u_{t}(x, t)-\operatorname{div}(\kappa(x) \nabla u(x, t))=0, \quad \text { in } \Omega \times(0, T],
$$

satisfying

$$
\begin{gathered}
u(\cdot, 0)=0, \quad \text { on } \bar{\Omega} \times\{0\}, \\
\kappa \nabla u \cdot \nu=0, \quad \text { on } \Gamma \times[0, T],
\end{gathered}
$$

where $\Gamma$ is an open portion of $\partial \Omega$ and $\kappa$ satisfies (3.1.1). Let $x_{0} \in \Gamma$ be such that

$$
d\left(x_{0}, \partial \Omega \backslash \Gamma\right)>R_{0} .
$$

For every $r_{1}, r_{2}, 0<r_{1}<r_{2}<2 r_{2} \leq \theta^{*} R_{0}$, we have

$$
\begin{gathered}
\int_{\Delta_{r_{2}}\left(x_{0}\right) \cap \Omega} u^{2}\left(x, t_{0}\right) d x \\
\leq \tilde{C}\left(\frac{R_{0}}{r_{2}}\right)^{C} \bar{H}^{2\left(1-\bar{\beta} \gamma^{\prime}\right)}\left(\int_{\Delta_{r_{1}}\left(x_{0}\right) \cap \Omega} u^{2}\left(x, t_{0}\right) d x\right)^{\bar{\beta} \gamma^{\prime}}, \quad \text { for every } t_{0} \in\left(0, \frac{T}{2}\right),
\end{gathered}
$$

$$
\int_{0}^{T / 2} \int_{\Delta_{r_{2}}\left(x_{0}\right) \cap \Omega} u^{2} d x d t \leq \tilde{C}\left(\frac{R_{0}}{r_{2}}\right)^{C}\left(T \bar{H}^{2}\right)^{1-\bar{\beta} \gamma^{\prime}}\left(\int_{0}^{T / 2} \int_{\Delta_{r_{1}}\left(x_{0}\right) \cap \Omega} u^{2} d x d t\right)^{\bar{\beta} \gamma^{\prime}}
$$

where

$$
\begin{gathered}
\gamma^{\prime}=\frac{\log \left(\frac{1}{2}+C \frac{R_{0}}{\tilde{r}_{2}}\right)}{\log \left(\frac{1}{2}+C \frac{R_{0}}{\tilde{r}_{2}}\right)+C \log \frac{C \tilde{r}_{2}}{r_{1}}}, \\
\tilde{r}_{2}=\mu 2 r_{2}+(1-\mu) 2 \theta^{*}\left(4 \max \left\{\sqrt{2}, \lambda_{2}^{\prime}\right\}\right) R_{0}, \\
\bar{H}=\sup _{t \in[0, T]}\|u(\cdot, t)\|_{H^{1}\left(\Omega \cap \Delta_{R_{0}}\right)},
\end{gathered}
$$

where $\mu, 0<\mu<1, \bar{\beta}, 0<\bar{\beta}<1, \theta^{*}, 0<\theta^{*}<1, \lambda_{2}^{\prime} \geq 1$ and $C>0$ depend on $\lambda$, $\Lambda$ and $E$ only, and $\tilde{C} \geq 1$ depends on $\lambda, \Lambda, E$ and $\frac{R_{0}^{2}}{T}$ only.

Proof of Theorem 3.2.1. First, let us assume that $\kappa\left(x_{0}\right)=I d$. We fix coordinates $\left(x^{\prime}, x_{n}\right)$ suitable for the local representation of the boundary as a graph as in Definition 2.1. Namely, we have $x_{0}=0$ and

$$
\Omega \cap \Delta_{R_{0}}(0)=\left\{x \in \Delta_{R_{0}}(0) \text { s.t. } x_{n}>\varphi\left(x^{\prime}\right)\right\},
$$

where $\varphi$ is a $C^{1,1}$ function on $\Delta_{R_{0}}^{\prime}(0) \subset \mathbb{R}^{n-1}$ satisfying

$$
\varphi(0)=|\nabla \varphi(0)|=0
$$

and

$$
\|\varphi\|_{C^{1,1}\left(\Delta_{R_{0}}^{\prime}(0)\right)} \leq E R_{0}
$$


For the reader's convenience, we recall the transformation flattening the boundary introduced in [AE], see also [AlBRV]. We can construct a map $\Phi \in C^{1,1}\left(\Delta_{R_{2}}(0), \mathbb{R}^{n}\right)$ such that

$$
\begin{gathered}
\Phi\left(\Delta_{R_{2}}(0)\right) \subset \Delta_{R_{1}}(0), \\
\Phi\left(y^{\prime}, 0\right)=\left(y^{\prime}, \varphi\left(y^{\prime}\right)\right), \quad \text { for every } y^{\prime} \in \Delta_{R_{2}}^{\prime}(0) \subset \mathbb{R}^{n-1}, \\
\Phi\left(\Delta_{R_{2}}^{+}(0)\right) \subset \Omega \cap \Delta_{R_{1}}(0), \\
\frac{1}{2}|y-z| \leq|\Phi(y)-\Phi(z)| \leq C_{1}^{\prime}|y-z|, \quad \text { for every } y, z \in \Delta_{R_{2}}(0), \\
\frac{1}{2^{n}} \leq|\operatorname{det} D \Phi| \leq C_{2}^{\prime}, \\
|\operatorname{det} D \Phi(y)-\operatorname{det} D \Phi(z)| \leq \frac{C_{3}^{\prime}}{R_{0}}|y-z|, \quad \text { for every } y, z \in \Delta_{R_{2}}(0),
\end{gathered}
$$

where $R_{i}=\bar{\theta}_{i} R_{0}, 0<\bar{\theta}_{i}<1, i=1,2$, and $C_{1}^{\prime}, C_{2}^{\prime}, C_{3}^{\prime}, \bar{\theta}_{1}, \bar{\theta}_{2}$ only depend on $\lambda, \Lambda$ and $E$. Denoting

$$
\begin{gathered}
\bar{\kappa}(y)=|\operatorname{det} D \Phi(y)|\left(D \Phi^{-1}\right)(\Phi(y)) \kappa(\Phi(y))\left(D \Phi^{-1}\right)^{T}(\Phi(y)), \\
v(y, t)=u(\Phi(y), t),
\end{gathered}
$$

we have

$$
\begin{gathered}
\bar{\kappa}(0)=I d, \\
\bar{\kappa}_{n k}\left(y^{\prime}, 0\right)=0, \quad \text { for } k=1, \ldots, n-1 .
\end{gathered}
$$

Moreover, we have that the ellipticity and Lipschitz constants $\lambda^{\prime}, \Lambda^{\prime}$ of $\bar{\kappa}$ in $\Delta_{R_{2}}^{+}(0)$ depend on $\lambda, \Lambda$ and $E$ only. For every $y \in \Delta_{R_{2}}(0)$, let us denote by $\kappa^{\prime}(y)$ the symmetric matrix whose entries are given by

$$
\begin{aligned}
& \kappa_{i j}^{\prime}\left(y^{\prime}, y_{n}\right)=\bar{\kappa}_{i j}\left(y^{\prime},\left|y_{n}\right|\right), \quad \text { if either } 1 \leq i, j \leq n-1, \text { or } i=j=n, \\
& \kappa_{n j}^{\prime}\left(y^{\prime}, y_{n}\right)=\kappa_{j n}^{\prime}\left(y^{\prime}, y_{n}\right)=\operatorname{sgn}\left(y_{n}\right) \bar{\kappa}_{j n}\left(y^{\prime},\left|y_{n}\right|\right), \quad \text { if } 1 \leq j \leq n-1 .
\end{aligned}
$$

We have that $\kappa^{\prime}$ satisfies the same ellipticity and Lipschitz continuity conditions as $\bar{\kappa}$.

Denoting

$$
\begin{gathered}
U(y, t)=v\left(y^{\prime},\left|y_{n}\right|, t\right), \quad \text { for } y \in \Delta_{R_{2}}(0), t \in(0, T), \\
b(y)=\left|\operatorname{det} D \Phi\left(y^{\prime},\left|y_{n}\right|\right)\right| \quad \text { for } y \in \Delta_{R_{2}}(0),
\end{gathered}
$$

we have that $U \in H^{2,1}\left(\Delta_{R_{2}}(0) \times(0, T)\right)$ is a solution to

$$
b(y) U_{t}(y, t)-\operatorname{div}\left(\kappa^{\prime}(y) \nabla U(y, t)\right)=0, \quad \text { in } \Delta_{R_{2}}(0) \times(0, T),
$$

and satisfies

$$
U(\cdot, 0)=0, \quad \text { on } \Delta_{R_{2}}(0) .
$$

Moreover, from (3.2.6d) we have that

$$
\Omega \cap \Delta_{r / 2}(0) \subset \Phi\left(\Delta_{r}^{+}(0)\right) \subset \Omega \cap \Delta_{C_{1}^{\prime} r}(0), \quad \text { for every } r \leq R_{2} .
$$




$$
\Delta_{r / C_{1}^{\prime}}^{+}(0) \subset \Phi^{-1}\left(\Omega \cap \Delta_{r}(0)\right) \subset \Delta_{2 r}^{+}(0), \quad \text { for every } r \leq R_{1} .
$$

Let us set $\theta^{*}=\frac{\left(4 \max \left\{\sqrt{2}, \lambda_{2}^{\prime}\right\}\right)^{-1} \bar{\theta} \bar{\theta}_{2}}{4}$, where $\bar{\theta}$ has been introduced in Theorem 3.1.1 and $\lambda_{2}^{\prime}=\max \left\{\lambda^{\prime}, C_{2}^{\prime}, 2^{n}\right\}$. Let $r_{1}, r_{2}$ be such that $0<r_{1}<r_{2}<2 r_{2} \leq \theta^{*} R_{0}$. By the choice made of $\theta^{*}$, we can apply (3.1.7a)-(3.1.7b) to $U$ for the triplet of radii $s_{1}=$ $\frac{r_{1}}{C_{1}^{\prime}}, s_{2}=2 r_{2}, s_{3}=\frac{\bar{\theta} R_{2}}{2}=2 \theta^{*}\left(4 \max \left\{\sqrt{2}, \lambda_{2}^{\prime}\right\}\right) R_{0}$. By simple changes of variables in the integrals we obtain (3.2.4) - (3.2.5). In the general case $\kappa\left(x_{0}\right) \neq I d$, we can consider a linear transformation $S: \mathbb{R}^{n} \rightarrow \mathbb{R}^{n}$ such that, setting $\tilde{\kappa}(S x)=\frac{S \kappa(x) S^{T}}{|\operatorname{det} S|}$, we have $\tilde{\kappa}\left(x_{0}\right)=I d$ (here, as above, we identify $x_{0}=0$ ). We have that, under such a transformation, the modified coefficient $\tilde{\kappa}$, the transformed domain $S(\Omega)$ and the transformed boundary portion $S(\Gamma)$ satisfy assumptions analogous to (3.1.1), (3.2.1) and (3.2.3) with constants which are dominated by the a priori constants $\lambda, \Lambda, R_{0}, E$, up to multiplicative factors which only depend on $\lambda$. Moreover, $\tilde{H}=\sup _{t \in[0, T]}\left\|\left(u \cdot S^{-1}\right)(\cdot, t)\right\|_{H^{1}\left(S\left(\Omega \cap \Delta_{R_{0}}\right)\right)}$ is dominated by $\bar{H}$ up to a multiplicative factor which only depends on $\lambda$. We also have that the ellipsoids $S\left(\Delta_{r}\left(x_{0}\right)\right)$ satisfy

$$
\Delta_{\frac{r}{\sqrt{\lambda}}}\left(x_{0}\right) \subset S\left(\Delta_{r}\left(x_{0}\right)\right) \subset \Delta_{\sqrt{\lambda} r}\left(x_{0}\right), \quad \text { for every } r>0 .
$$

Therefore, by a change of variables, using the result just proved when $\kappa\left(x_{0}\right)=I d$, the thesis follows.

Theorem 3.2.1'. (Three Spheres Inequalities and Three Cylinders Inequalities at the Boundary). Let the hypotheses of Theorem 3.2.1, except (3.2.2b), be satisfied. For every $r_{1}, r_{2}$ such that $0<r_{1}<r_{2}<2 r_{2} \leq \theta^{*} R_{0}$, we have

$$
\begin{gathered}
\int_{\Delta_{r_{2}}\left(x_{0}\right) \cap \Omega} u^{2}\left(x, t_{0}\right) d x \\
\leq \tilde{C}_{1}\left(\frac{R_{0}}{r_{2}}\right)^{C} \bar{H}^{2\left(1-\bar{\beta} \gamma^{\prime}\right)}\left(\int_{\Delta_{r_{1}}\left(x_{0}\right) \cap \Omega} u^{2}\left(x, t_{0}\right) d x\right)^{\bar{\beta} \gamma^{\prime}} \quad, \quad \text { for every } t_{0} \in(0, T),
\end{gathered}
$$

where $\gamma^{\prime}$ is given by (3.2.5a)-(3.2.5b), $\bar{H}$ is given by (3.2.5c), $\bar{\beta}, 0<\bar{\beta}<1, \lambda_{2}^{\prime} \geq 1$ and $C>0$ depend on $\lambda, \Lambda$ and $E$ only, $\theta^{*}, 0<\theta^{*}<1$, depends on $\lambda, \Lambda, E$ and $\frac{t_{0}}{R_{0}^{2}}$ only, $\theta^{*}=O\left(t_{0}\right)$ as $t_{0} \rightarrow 0$, and $\tilde{C}_{1}$ depends on $\lambda, \Lambda, E, \frac{R_{0}^{2}}{T}$ and $\frac{T}{T-t_{0}}$ only. Let $\sigma \in\left(0, \frac{1}{2}\right)$. For every $r_{1}, r_{2}$ such that $0<r_{1}<r_{2}<2 r_{2} \leq \tilde{\theta} R_{0}$, we have

$$
\begin{gathered}
\int_{\sigma T}^{(1-\sigma) T} \int_{\Delta_{r_{2}}\left(x_{0}\right) \cap \Omega} u^{2}(x, t) d x d t \\
\leq \tilde{C}_{2}\left(\frac{R_{0}}{r_{2}}\right)^{C}\left(T \bar{H}^{2}\right)^{1-\bar{\beta} \gamma^{\prime}}\left(\int_{\sigma T}^{(1-\sigma) T} \int_{\Delta_{r_{1}}\left(x_{0}\right) \cap \Omega} u^{2}(x, t) d x d t\right)^{\bar{\beta} \gamma^{\prime}},
\end{gathered}
$$

where $\bar{H}, \gamma^{\prime}, \bar{\beta}$ and $C$ are as above, $\tilde{\theta}, 0<\tilde{\theta}<1$, and $\tilde{C}_{2}$ depend on $\lambda, \Lambda, E, \sigma$ and $\frac{R_{0}^{2}}{T}$ only.

Proof. The proof follows by slight changes of the proof of Theorem 3.2.1, with

$$
\theta^{*}=\left(4 \max \left\{\sqrt{2}, \lambda_{2}^{\prime}\right\}\right)^{-1} \min \left\{\frac{t_{0}}{\lambda_{2}^{\prime} \sqrt{c_{P}} \bar{\theta}_{2} R_{0}^{2}}, \frac{\bar{\theta} \bar{\theta}_{2}}{4}\right\},
$$




$$
\tilde{\theta}=\left(4 \max \left\{\sqrt{2}, \lambda_{2}^{\prime}\right\}\right)^{-1} \min \left\{\frac{\sigma T}{\lambda_{2}^{\prime} \sqrt{c_{P}} \bar{\theta}_{2} R_{0}^{2}}, \frac{\bar{\theta} \bar{\theta}_{2}}{4}\right\},
$$

in the notation of the proof of Theorem 3.2.1.

\section{Subsection 3.3 (Stability Estimates of Continuation from Cauchy Data on Time-like Surfaces).}

Theorem 3.3.1. (Stability Estimates of Continuation from Cauchy Data on Timelike Surfaces). Let $\Omega$ be a domain satisfying (3.2.1). Let $\Sigma$ be an open portion of $\partial \Omega$ satisfying

$$
\partial \Omega \cap \Delta_{R_{0}}\left(P_{1}\right) \subset \Sigma,
$$

for some $P_{1} \in \Sigma$. Let $u \in H^{2,1}(\Omega \times(0, T))$ be a solution to (3.2.2a), (3.2.2b) satisfying

$$
\begin{gathered}
\|u\|_{H^{3 / 2,3 / 4}(\Sigma \times(0, T))} \leq T^{\frac{1}{2}} R_{0}^{\frac{n-1}{2}} \bar{\epsilon} \\
R_{0}\left\|\frac{\partial u}{\partial \nu}\right\|_{H^{1 / 2,1 / 4}(\Sigma \times(0, T))} \leq T^{\frac{1}{2}} R_{0}^{\frac{n-1}{2}} \bar{\epsilon} .
\end{gathered}
$$

For every $t_{0} \in[0, T / 2]$ we have

$$
\left\|u\left(\cdot, t_{0}\right)\right\|_{L^{2}\left(\Delta_{\tilde{\theta}_{R_{0}}}\left(P_{2}\right)\right)} \leq C R_{0}^{\frac{n}{2}}\left(T^{-\frac{1}{2}} R_{0}^{-\frac{n}{2}}\|u\|_{H^{2,1}(\Omega \times(0, T))}\right)^{1-\bar{\gamma}} \bar{\epsilon}^{\bar{\gamma}},
$$

where $P_{2}=P_{1}-\tilde{\theta} R_{0} \nu, \nu$ denotes the outer unit normal to $\Omega$ at $P_{1}, \bar{\gamma}, 0<\bar{\gamma}<1$, depends on $\lambda$ and $\Lambda$ only, $\tilde{\theta}, 0<\tilde{\theta}<\frac{1}{2}$, depends on $\lambda$ and $E$ only, $C \geq 1$ depends on $\lambda, \Lambda, E$ and $\frac{R_{0}^{2}}{T}$ only.

Proof of Theorem 3.3.1. We may assume that $R_{0}^{n / 2} T^{1 / 2} \bar{\epsilon} \leq\|u\|_{H^{2,1}(\Omega \times(0, T))}$, since, otherwise, (3.3.4) is trivial. Let us denote

$$
\begin{gathered}
G=\Omega \cap \Delta_{R_{0}}\left(P_{1}\right), \\
\Sigma_{0}=\partial \Omega \cap \Delta_{R_{0}}\left(P_{1}\right), \\
Q=\Delta_{R_{0}}\left(P_{1}\right) \times(0, T), \\
Q^{+}=G \times(0, T), \\
Q^{-}=\left(\Delta_{R_{0}}\left(P_{1}\right) \backslash \bar{G}\right) \times(0, T) .
\end{gathered}
$$

Up to a rigid motion, we have

$$
\begin{gathered}
P_{1}=0, \\
G=\left\{x=\left(x^{\prime}, x_{n}\right) \in \Delta_{R_{0}} \text { s.t. } x_{n}>\varphi\left(x^{\prime}\right)\right\}, \\
\Sigma_{0}=\left\{x=\left(x^{\prime}, x_{n}\right) \in \Delta_{R_{0}} \text { s.t. } x_{n}=\varphi\left(x^{\prime}\right)\right\},
\end{gathered}
$$

where $\varphi$ is a $C^{1,1}$ function on $\Delta_{R_{0}} \subset \mathbb{R}^{n-1}$ satisfying

$$
\varphi(0)=|\nabla \varphi(0)|=0
$$

and

$$
\|\varphi\|_{C^{1,1}\left(\Delta_{R_{0}}\right)} \leq E R_{0}
$$


By extension theorems in Sobolev spaces [LioM], there exists $v \in H^{2,1}\left(Q^{+}\right)$such that

$$
\begin{gathered}
v=u, \quad \frac{\partial v}{\partial \nu}=\frac{\partial u}{\partial \nu}, \quad \text { on } \Sigma_{0} \\
\|v\|_{H^{2,1}\left(Q^{+}\right)} \leq C R_{0}^{\frac{n}{2}} T^{\frac{1}{2}} \bar{\epsilon}
\end{gathered}
$$

with $C$ only depending on $E$ and $\frac{R_{0}^{2}}{T}$. The function $w=u-v \in H^{2,1}\left(Q^{+}\right)$satisfies

$$
\begin{gathered}
L w=-L v, \quad \text { in } Q^{+}, \\
w=0, \quad \frac{\partial w}{\partial \nu}=0, \quad \text { on } \Sigma_{0} \times(0, T),
\end{gathered}
$$

where $L$ denotes the operator

$$
L \cdot=\frac{\partial \cdot}{\partial t}-\operatorname{div}(\kappa \nabla \cdot)
$$

Let us define

$$
\begin{aligned}
& \bar{f}=\left\{\begin{array}{cc}
L v & \text { in } Q^{+}, \\
0 & \text { in } Q^{-},
\end{array}\right. \\
& \bar{w}= \begin{cases}w & \text { in } Q^{+}, \\
0 & \text { in } Q^{-} .\end{cases}
\end{aligned}
$$

Since $w=\frac{\partial w}{\partial \nu}=0$ on $\Sigma_{0} \times(0, T)$, we have that $\bar{w} \in H^{2,1}(Q)$ and $L \bar{w}=-\bar{f}$ in $Q$. Let $\bar{z} \in H^{2,1}(Q)$ be the solution to

$$
\begin{gathered}
L \bar{z}=-\bar{f}, \\
\left.\bar{z}\right|_{\partial_{P} Q}=0,
\end{gathered}
$$

where $\partial_{P} Q$ denotes the parabolic boundary of $Q$. We have that $L(\bar{w}-\bar{z})=0$ in $Q$. By the regularity of $\varphi, G$ satisfies an interior and exterior sphere condition at $P_{1}=0$. More precisely, setting $R_{1}=\min \left\{\frac{1}{2}, \frac{1}{E}\right\} R_{0}, P_{r}=r e_{n}, Q_{r}=-r e_{n}$, we have that

$$
\Delta_{r}\left(P_{r}\right) \subset G, \quad \Delta_{r}\left(Q_{r}\right) \subset \Delta_{R_{0}} \backslash \bar{G}, \quad \text { for every } r \leq R_{1} .
$$

Since $L(\bar{w}-\bar{z})=0$ in $\Delta_{R_{0}-R_{1}}\left(Q_{r}\right) \times(0, T)$, for every $r \leq R_{0}-R_{1}$, we can apply (3.1.7a), making the following positions: $r_{1}=r, r_{2}=3 r, r_{3}=13 \max \left\{\sqrt{2}, \lambda_{2}\right\} r$. For every $r \leq \tilde{\theta} R_{0}$ and for every $t_{0} \in(0, T / 2)$ we have

$$
\int_{\Delta_{3 r}\left(Q_{r}\right)}(\bar{w}-\bar{z})^{2}\left(x, t_{0}\right) d x \leq C H^{2(1-\bar{\gamma})}\left(\int_{\Delta_{r}\left(Q_{r}\right)}(\bar{w}-\bar{z})^{2}\left(x, t_{0}\right) d x\right)^{\bar{\gamma}},
$$

where $H=\sup _{t \in[0, T]}\|(\bar{w}-\bar{z})(\cdot, t)\|_{H^{1}\left(\Delta_{R_{0}}\right)}, \tilde{\theta}=\frac{\bar{\theta} \max \{1 / 2,1-(1 / E)\}}{26 \max \left\{\sqrt{2}, \lambda_{2}\right\}}$ depends on $\lambda$, $\Lambda$ and $E$ only, with $\bar{\theta}$ introduced in Theorem 3.1.1, $\bar{\gamma}, 0<\bar{\gamma}<1$, depends on $\lambda$ only, $C \geq 1$ depends on $\lambda, \Lambda, E$ and $\frac{R_{0}^{2}}{T}$ only. Since $\Delta_{r}\left(P_{r}\right) \subset \Delta_{3 r}\left(Q_{r}\right)$, choosing $r=\tilde{\theta} R_{0}$ and recalling the definition of $w$, we have

$$
\left\|(w-\bar{z})\left(\cdot, t_{0}\right)\right\|_{L^{2}\left(\Delta_{\tilde{\theta} R_{0}}\left(P_{\tilde{\theta} R_{0}}\right)\right)}^{2} \leq C H^{2(1-\bar{\gamma})}\left\|\bar{z}\left(\cdot, t_{0}\right)\right\|_{L^{2}\left(\Delta_{\tilde{\theta} R_{0}}\left(Q_{\tilde{\theta} R_{0}}\right)\right)}^{2 \bar{y}},
$$


where $C$ depends on $\lambda, \Lambda, E$ and $\frac{R_{0}^{2}}{T}$ only. We have

$$
\begin{gathered}
\|\bar{f}\|_{L^{2}(Q)}=\|L v\|_{L^{2}\left(Q^{+}\right)} \leq C\|v\|_{H^{2,1}\left(Q^{+}\right)}, \\
\|\bar{z}\|_{H^{2,1}(Q)} \leq\|\bar{f}\|_{L^{2}(Q)},
\end{gathered}
$$

where $C$ depends on $\lambda$ only. From (3.3.5b), (3.3.8), (3.3.9) and the triangle inequality we have

$$
\|\bar{w}-\bar{z}\|_{H^{2,1}(Q)} \leq C\|u\|_{H^{2,1}(\Omega \times(0, T))},
$$

where $C$ depends on $E, \lambda$ and $\frac{R_{0}^{2}}{T}$ only. By using trace inequalities, we have

$$
\left\|(\bar{w}-\bar{z})\left(\cdot, t_{0}\right)\right\|_{H^{1}\left(\Delta_{R_{0}}\right)} \leq C T^{-\frac{1}{2}}\|\bar{w}-\bar{z}\|_{H^{2,1}(Q)},
$$

where $C$ depends on $E$ and $\frac{R_{0}^{2}}{T}$ only, so that, by (3.3.10), we obtain

$$
H \leq C T^{-\frac{1}{2}}\|u\|_{H^{2,1}(\Omega \times(0, T))},
$$

with $C$ depending on $\lambda, E$ and $\frac{R_{0}^{2}}{T}$ only. From (3.3.5b), (3.3.7) - (3.3.12) we obtain

$$
\left\|(w-\bar{z})\left(\cdot, t_{0}\right)\right\|_{L^{2}\left(\Delta_{\tilde{\theta} R_{0}}\left(P_{\tilde{\theta} R_{0}}\right)\right.} \leq C\left(R_{0}^{\frac{n}{2}} \bar{\epsilon}\right)^{\bar{\gamma}}\left(T^{-\frac{1}{2}}\|u\|_{H^{2,1}(\Omega \times(0, T))}\right)^{1-\bar{\gamma}},
$$

where $C$ depends on $\lambda, \Lambda, E$ and $\frac{R_{0}^{2}}{T}$ only. From (3.3.5b), (3.3.8), (3.3.9), (3.3.13), the triangle inequality and trace inequalities, we have, for every $t_{0} \in[0, T / 2]$,

$$
\left\|u\left(\cdot, t_{0}\right)\right\|_{L^{2}\left(\Delta_{\tilde{\theta} R_{0}}\left(P_{\tilde{\theta} R_{0}}\right)\right.} \leq C\left(R_{0}^{\frac{n}{2}} \bar{\epsilon}\right)^{\bar{\gamma}}\left(T^{-\frac{1}{2}}\|u\|_{H^{2,1}(\Omega \times(0, T))}\right)^{1-\bar{\gamma}},
$$

where $C$ depends on $\lambda, \Lambda, E$ and $\frac{R_{0}^{2}}{T}$ only. Now (3.3.4) follows.

\section{The Inverse Problem: the Main Result}

In this section $\Omega$ will be a bounded domain in $\mathbb{R}^{n}$, a part of which, say $I$ (perhaps some interior connected component of $\partial \Omega$ or some inaccessible portion of the exterior component of $\partial \Omega$ ), is not known. Let $A=\partial \Omega \backslash I$ be the accessible part of the boundary. Given a nontrivial function $g$ on $A \times(0, T)$, let us consider the parabolic boundary value problem

$$
\begin{gathered}
u_{t}(x, t)-\operatorname{div}(\kappa(x) \nabla u(x, t))=0, \quad \text { in } \Omega \times(0, T], \\
u=0 \quad \text { on } \bar{\Omega} \times\{0\}, \\
\kappa \nabla u \cdot \nu=0, \quad \text { on } I \times[0, T], \\
\kappa \nabla u \cdot \nu=g, \quad \text { on } A \times[0, T],
\end{gathered}
$$

where $\nu$ denotes the exterior unit normal to $\Omega$ and $\kappa$ is a function from $\mathbb{R}^{n}$ with values $n \times n$ symmetric matrices satisfying Lipschitz and uniform ellipticity conditions (see (4.8) below).

Given an open subset $\Sigma$ of the boundary of $\Omega$ which is contained in $A$, we consider the inverse problem of determining $I$ from the knowledge of $\kappa \nabla u \cdot \nu$ on $\Sigma \times[0, T]$.

i) A priori information on the domain.

Given $R_{0}, M>0$, we assume

$$
|\Omega| \leq M R_{0}^{n}
$$


Here, and in the sequel, $|\Omega|$ denotes the Lebesgue measure of $\Omega$. We shall distinguish two nonempty parts, $A, I$ in $\partial \Omega$, and we assume

$$
I \cup A=\partial \Omega, \quad \stackrel{\circ}{I} \cap \stackrel{\circ}{A}=\emptyset, \quad I \cap A=\partial A=\partial I .
$$

Here, interiors and boundaries are intended in the relative topology in $\partial \Omega$. Moreover we assume that we can select a portion $\Sigma$ within $A$ satisfying, for some $P_{1} \in \Sigma$,

$$
\partial \Omega \cap \Delta_{R_{0}}\left(P_{1}\right) \subset \Sigma,
$$

and also, denoting by $I^{R_{0}}$ the portion of $\partial \Omega$ of all $x \in \partial \Omega \operatorname{such}$ that $\operatorname{dist}(x, I)<R_{0}$,

$$
\Sigma \cap I^{R_{0}}=\emptyset \text {. }
$$

Regarding the regularity of $\partial \Omega$, we assume that, given $E>0$,

$$
\partial \Omega \text { is of class } C^{1,1} \text { with constants } R_{0}, E \text {. }
$$

Remark 4.1. Observe that (4.6) automatically implies a lower bound on the diameter of every connected component of $\partial \Omega$. Moreover, by combining (4.2) with (4.6), an upper bound on the diameter of $\Omega$ can also be obtained. Note also that (4.2), (4.6) implicitly comprise an a priori upper bound on the number of connected components of $\partial \Omega$.

ii) Assumptions about the boundary data.

Let us set

$$
A_{R_{0}}=\left\{x \in A \text { s.t. } \operatorname{dist}(x, I) \geq R_{0}\right\},
$$

(that is: $A_{R_{0}}=\partial \Omega \backslash I^{R_{0}}$ ). Denoting again by $g$ the extension by 0 of the Neumann data $g$ appearing in problem (4.1) to $S_{T}=\partial \Omega \times[0, T]$, we shall assume

$$
\begin{gathered}
g \in H^{1 / 2,1 / 4}\left(S_{T}\right), \quad g \not \equiv 0, \\
\operatorname{supp} g \subset A_{R_{0}} \times[0, T],
\end{gathered}
$$

and, for a given constant $F>0$,

$$
\frac{\|g\|_{H^{1 / 2,1 / 4}\left(S_{T}\right)}}{\|g\|_{L^{2}\left(S_{T}\right)}} \leq F .
$$

iii) Assumptions about the thermal conductivity $\kappa$.

The thermal conductivity $\kappa$ is assumed to be a given function from $\mathbb{R}^{n}$ with values $n \times n$ symmetric matrices satisfying the following conditions for given constants $\lambda$, $\Lambda, \lambda \geq 1, \Lambda \geq 0$,

$$
\begin{gathered}
\lambda^{-1}|\xi|^{2} \leq \kappa(x) \xi \cdot \xi \leq \lambda|\xi|^{2}, \quad \text { for every } x, \xi \in \mathbb{R}^{n} \quad \text { (ellipticity), } \\
|\kappa(x)-\kappa(y)| \leq \Lambda \frac{|x-y|}{R_{0}}, \quad \text { for every } x, y \in \mathbb{R}^{n} \quad \text { (Lipschitz continuity). }
\end{gathered}
$$

In the sequel, we shall refer to the set of constants $\lambda, \Lambda, E, M, \frac{R_{0}^{2}}{T}, F$, as the $a$ priori data.

Theorem 4.1. Let $\Omega_{1}, \Omega_{2}$ be two domains satisfying (4.2), (4.6). Let $A_{i}, I_{i}, i=$ 1,2 , be the corresponding accessible and inaccessible parts of their boundaries. Let us assume that $A_{1}=A_{2}=A, \Omega_{1}, \Omega_{2}$ lie on the same side of $A$, and that (4.3)-(4.5) 
are satisfied by both pairs $A_{i}, I_{i}$. Let $u_{i} \in H^{2,1}\left(\Omega_{i} \times(0, T)\right)$ be the solution to (4.1) when $\Omega=\Omega_{i}, i=1,2$, and let (4.7) and (4.8) be satisfied. If, given $\epsilon>0$, we have

$$
\left\|u_{1}-u_{2}\right\|_{L^{2}(\Sigma \times(0, T))} \leq T^{\frac{1}{2}} R_{0}^{\frac{n-1}{2}} \epsilon
$$

then we have

$$
d_{\mathcal{H}}\left(\bar{\Omega}_{1}, \bar{\Omega}_{2}\right) \leq R_{0} \omega\left(\frac{T^{\frac{1}{2}} R_{0}^{\frac{n-3}{2}} \epsilon}{\|g\|_{H^{1 / 2,1 / 4}\left(S_{T}\right)}}\right),
$$

where $\omega$ is an increasing continuous function on $[0, \infty)$ which satisfies

$$
\omega(t) \leq C|\log t|^{-B}, \quad \text { for every } t<1,
$$

and $C, B$ are positive constants only depending on the a priori data.

Here $d_{\mathcal{H}}$ denotes the Hausdorff distance between bounded closed sets of $\mathbb{R}^{n}$.

\section{Proof of Theorem 4.1}

Here and in the sequel we shall denote by $G$ the connected component of $\Omega_{1} \cap \Omega_{2}$ such that $\Sigma \subset \bar{G}$.

The proof of Theorem 4.1 is obtained from the following sequence of propositions.

Proposition 5.1. (Three Cylinders Inequalities in the Interior). Let $\Omega$ be a domain satisfying (4.2), such that $\partial \Omega$ is of class $C^{1,1}$ with constants $R_{0}, E$. Let $u \in H^{2,1}(\Omega \times(0, T))$ be the solution to (4.1), where $g$ satisfies (4.7a) and $\kappa$ satisfies (4.8). For every $\rho>0$ and every $x_{0} \in \Omega_{\rho}$, we have

$$
\begin{gathered}
\int_{0}^{T / 2} \int_{\Delta_{r_{2}}\left(x_{0}\right)} u^{2}(x, t) d x d t \leq \tilde{C} \frac{\rho}{\rho-r_{2}}\left(\frac{\rho}{r_{2}}\right)^{C} \\
\times\left(R_{0}^{3}\left(1+\frac{T^{2}}{\rho^{4}}\right)\|g\|_{H^{\frac{1}{2}, \frac{1}{4}\left(S_{T}\right)}}^{2}\right)^{1-\bar{\beta} \gamma^{\prime}}\left(\int_{0}^{T / 2} \int_{\Delta_{r_{1}}\left(x_{0}\right)} u^{2}(x, t) d x d t\right)^{\bar{\beta} \gamma^{\prime}}
\end{gathered}
$$

for every $r_{1}, r_{2}$ such that $0<r_{1}<r_{2}<\theta \rho$, where

$$
\begin{gathered}
\gamma^{\prime}=\frac{\log \left(\frac{1}{2}+\frac{(4 \max \{\sqrt{2}, \lambda\}) \theta \rho}{2 \lambda r_{2}^{\prime}}\right)}{\log \left(\frac{1}{2}+\frac{(4 \max \{\sqrt{2}, \lambda\}) \theta \rho}{2 \lambda r_{2}^{\prime}}\right)+C \log \frac{2 \lambda r_{2}^{\prime}}{a r_{1}},} \\
r_{2}^{\prime}=\frac{4 \lambda-2}{4 \lambda-1} r_{2}+\frac{1}{4 \lambda-1}(4 \max \{\sqrt{2}, \lambda\}) \theta \rho, \\
a=\frac{3}{4 \sqrt{2} e \pi \lambda},
\end{gathered}
$$

where $\bar{\beta}, 0<\bar{\beta}<1$, depends on $\lambda$ only, $\theta, 0<\theta<1$, and $C>0$ depend on $\lambda$ and $\Lambda$ only, and $\tilde{C} \geq 1$ depends on $\lambda, \Lambda, E$ and $\frac{R_{0}^{2}}{T}$ only.

Proposition 5.2. (Three Cylinders Inequalities at the Boundary). Let $\Omega$ be $a$ domain satisfying (4.2) and (4.6). Let us assume that the accessible and inaccessible parts $A, I$ of its boundary satisfy $(4.3)-(4.5)$. Let $u \in H^{2,1}(\Omega \times(0, T))$ be the 
solution to (4.1) and let (4.7) and (4.8) be satisfied. Let $x_{0} \in I$. For every $r_{1}, r_{2}$, $0<r_{1}<r_{2}<2 r_{2} \leq \theta^{*} R_{0}$, we have

$$
\begin{gathered}
\int_{0}^{T / 2} \int_{\Delta_{r_{2}}\left(x_{0}\right) \cap \Omega} u^{2}(x, t) d x d t \\
\leq \tilde{C}\left(\frac{R_{0}}{r_{2}}\right)^{C}\left(R_{0}^{3}\|g\|_{H^{\frac{1}{2}, \frac{1}{4}\left(S_{T}\right)}}^{2}\right)^{1-\bar{\beta} \gamma^{\prime}}\left(\int_{0}^{T / 2} \int_{\Delta_{r_{1}}\left(x_{0}\right) \cap \Omega} u^{2}(x, t) d x d t\right)^{\bar{\beta} \gamma^{\prime}},
\end{gathered}
$$

where

$$
\begin{gathered}
\gamma^{\prime}=\frac{\log \left(\frac{1}{2}+C \frac{R_{0}}{\tilde{r}_{2}}\right)}{\log \left(\frac{1}{2}+C \frac{R_{0}}{\tilde{r}_{2}}\right)+C \log \frac{C \tilde{r}_{2}}{r_{1}}}, \\
\tilde{r}_{2}=\mu 2 r_{2}+(1-\mu) 2 \theta^{*}\left(4 \max \left\{\sqrt{2}, \lambda_{2}\right\}\right) R_{0},
\end{gathered}
$$

where $\mu, 0<\mu<1, \bar{\beta}, 0<\bar{\beta}<1, \theta^{*}, 0<\theta^{*}<1$, and $C>0$ depend on $\lambda, \Lambda$ and $E$ only, and $\tilde{C} \geq 1$ depends on $\lambda, \Lambda, E$ and $\frac{R_{0}^{2}}{T}$ only.

Proposition 5.3. (Stability Estimate of Continuation from Cauchy Data). Let the hypotheses of Theorem 4.1 be satisfied. We have

$$
\int_{0}^{T / 2} \int_{\Omega_{i} \backslash G} u_{i}^{2}(x, t) d x d t \leq R_{0}^{3}\|g\|_{H^{1 / 2,1 / 4}\left(S_{T}\right)}^{2} \omega\left(\frac{R_{0}^{\frac{n-3}{2}} T^{\frac{1}{2}} \epsilon}{\|g\|_{H^{1 / 2,1 / 4}\left(S_{T}\right)}}\right), \quad i=1,2,
$$

where $\omega$ is an increasing continuous function on $[0, \infty)$ which satisfies

$$
\omega(t) \leq C(\log |\log t|)^{-1 / n}, \quad \text { for every } t<e^{-1},
$$

and $C>0$ depends on $\lambda, \Lambda, E, M$ and $\frac{R_{0}^{2}}{T}$ only.

Proposition 5.4. (Improved Stability Estimate of Continuation from Cauchy Data). Let the hypotheses of Proposition 5.3 hold and, in addition, let us assume that there exist $L>0$ and $r_{0}, 0<r_{0} \leq R_{0}$, such that $\partial G$ is of Lipschitz class with constants $r_{0}, L$. Then (5.5) holds with $\omega$ given by

$$
\omega(t) \leq C|\log t|^{-B}, \quad \text { for every } t<1,
$$

where $B>0$ and $C>0$ depend only on $\lambda, \Lambda, E, M, \frac{R_{0}^{2}}{T}, L$ and $R_{0} / r_{0}$.

Proposition 5.5. (Stability Estimate of Continuation from the Interior). Let $\Omega$ be a domain in $\mathbb{R}^{n}$ satisfying (4.2), such that $\partial \Omega$ is of class $C^{1,1}$ with constants $R_{0}$, E. Let $u \in H^{2,1}(\Omega \times(0, T))$ be the solution to (4.1), where $g$ satisfies (4.7a), (4.7c) and $\kappa$ satisfies (4.8). For every $\rho>0$ and every $x_{0} \in \Omega_{\rho}$, we have

$$
\int_{0}^{T / 2} \int_{B_{\rho}\left(x_{0}\right)} u^{2} d x d t \geq C R_{0}^{3}\|g\|_{H^{1 / 2,1 / 4}\left(S_{T}\right)}^{2},
$$

where $C>0$ depends on $\lambda, \Lambda, E, M, \frac{R_{0}^{2}}{T}, F$ and $R_{0} / \rho$ only.

At this stage, we recall the notion of modified distance introduced in [AlBRV]. 
Definition 5.1. The modified distance between $\Omega_{1}$ and $\Omega_{2}$ is the number

$$
d_{m}\left(\Omega_{1}, \Omega_{2}\right)=\max \left\{\sup _{x \in \partial \Omega_{1}} \operatorname{dist}\left(x, \Omega_{2}\right), \sup _{x \in \partial \Omega_{2}} \operatorname{dist}\left(x, \Omega_{1}\right)\right\} .
$$

Notice that we obviously have

$$
d_{m}\left(\Omega_{1}, \Omega_{2}\right) \leq d_{\mathcal{H}}\left(\bar{\Omega}_{1}, \bar{\Omega}_{2}\right),
$$

but, in general, $d_{m}$ does not dominate the Hausdorff distance, and indeed it does not satisfy the axioms of a distance function. This is made clear by the following example: $\Omega_{1}=\Delta_{1}(0), \Omega_{2}=\Delta_{1}(0) \backslash \overline{\Delta_{1 / 2}(0)}$. In this case $d_{m}\left(\Omega_{1}, \Omega_{2}\right)=0$, whereas $d_{\mathcal{H}}\left(\Omega_{1}, \Omega_{2}\right)=1 / 2$.

The proof of the following proposition is given in AlBRV].

Proposition 5.6 (Geometric Lemma). Let $\Omega_{1}, \Omega_{2}$ be bounded domains satisfying (4.6). There exist numbers $d_{0}, r_{0}, d_{0}>0,0<r_{0} \leq R_{0}$, for which the ratios $\frac{d_{0}}{R_{0}}$, $\frac{r_{0}}{R_{0}}$ only depend on $E$, such that if we have

$$
d_{\mathcal{H}}\left(\bar{\Omega}_{1}, \bar{\Omega}_{2}\right) \leq d_{0},
$$

then the following facts hold:

i) There exists an absolute constant $C>0$ such that

$$
d_{\mathcal{H}}\left(\bar{\Omega}_{1}, \bar{\Omega}_{2}\right) \leq C d_{m}\left(\Omega_{1}, \Omega_{2}\right) .
$$

ii) Any connected component $G$ of $\Omega_{1} \cap \Omega_{2}$ has boundary of Lipschitz class with constants $r_{0}, L$, where $r_{0}$ is as above and $L>0$ only depends on $E$.

Proof of Theorem 4.1. Let us denote, for brevity, $d=d_{\mathcal{H}}\left(\bar{\Omega}_{1}, \bar{\Omega}_{2}\right), d_{m}=d_{m}\left(\Omega_{1}, \Omega_{2}\right)$ the modified distance defined by (5.9).

Let us prove that if $\eta>0$ is such that

$$
\int_{0}^{T / 2} \int_{\Omega_{i} \backslash G} u_{i}^{2}(x, t) d x d t \leq \eta, \quad i=1,2,
$$

then

$$
\begin{gathered}
d_{m} \leq C R_{0}\left(\frac{\eta}{R_{0}^{3}\|g\|_{H^{1 / 2,1 / 4}\left(S_{T}\right)}^{2}}\right)^{K}, \\
d \leq C R_{0}\left(\frac{\eta}{R_{0}^{3}\|g\|_{H^{1 / 2,1 / 4}\left(S_{T}\right)}^{2}}\right)^{K},
\end{gathered}
$$

where $C>0$ and $K>0$ depend on $\lambda, \Lambda, E, M, \frac{R_{0}^{2}}{T}$ and $F$ only. First, let us prove (5.14). We may assume, with no loss of generality, that there exists $x_{0} \in I_{1} \subset \partial \Omega_{1}$ such that $\operatorname{dist}\left(x_{0}, \Omega_{2}\right)=d_{m}$. By (5.13) we have

$$
\int_{0}^{T / 2} \int_{\Omega_{1} \cap \Delta_{d_{m}}\left(x_{0}\right)} u_{1}^{2}(x, t) d x d t \leq \eta .
$$

Two cases may occur:

I) $d_{m} \geq \frac{\theta^{*} R_{0}}{2}$,

II) $d_{m}<\frac{\theta^{*} R_{0}}{2}$, 
where $\theta^{*}$ has been introduced in Proposition 5.2.

If case I) occurs, let $\bar{d}=\frac{\theta^{*} R_{0}}{2\left(1+\sqrt{1+E^{2}}\right)}, \bar{x}=x_{0}-\sqrt{1+E^{2}} \bar{d} \nu$, where $\nu$ denotes the outer unit normal to $\Omega_{1}$ at $x_{0}$. We have that

$$
\Delta_{\bar{d}}(\bar{x}) \subset \Omega_{1} \cap \Delta_{\frac{\theta^{*} R_{0}}{2}}\left(x_{0}\right) .
$$

By (5.8) of Proposition 5.5 and by (5.17) we have

$$
\int_{0}^{T / 2} \int_{\Omega_{1} \cap \Delta_{\frac{\theta^{*} R_{0}}{2}}\left(x_{0}\right)} u_{1}^{2}(x, t) d x d t \geq C R_{0}^{3}\|g\|_{H^{1 / 2,1 / 4}\left(S_{T}\right)}^{2}
$$

where $C>0$ depends on $\lambda, \Lambda, E, M, \frac{R_{0}^{2}}{T}$ and $F$ only. On the other hand, it is evident that

$$
d_{m} \leq C R_{0}
$$

with $C$ depending on $E$ and $M$ only, so that, from (5.16), (5.18), (5.19), and from $d_{m} \geq \frac{\theta^{*} R_{0}}{2}$, we obtain

$$
d_{m} \leq C R_{0}\left(\frac{\eta}{R_{0}^{3}\|g\|_{H^{1 / 2,1 / 4}\left(S_{T}\right)}^{2}}\right)^{1 / 3}
$$

where $C$ depends on $\lambda, \Lambda, E, M, \frac{R_{0}^{2}}{T}$ and $F$ only.

If case II) occurs, let us apply (5.3b) of Proposition 5.2 with $r_{1}=d_{m}, r_{2}=\frac{\theta^{*} R_{0}}{2}$, obtaining, by (5.16),

$$
\int_{0}^{T / 2} \int_{\Omega_{1} \cap \Delta_{\frac{\theta^{*} R_{0}}{2}}\left(x_{0}\right)} u_{1}^{2}(x, t) d x d t \leq C R_{0}^{3}\|g\|_{H^{1 / 2,1 / 4}\left(S_{T}\right)}^{2}\left(\frac{\eta}{R_{0}^{3}\|g\|_{H^{1 / 2,1 / 4}\left(S_{T}\right)}^{2}}\right)^{\bar{\beta} \gamma^{\prime}}
$$

where $C$ depends on $\lambda, \Lambda, E$ and $\frac{R_{0}^{2}}{T}$ only and, moreover,

$$
\bar{\beta} \gamma^{\prime} \geq \frac{C}{\log \frac{R_{0}}{d_{m}}}
$$

with $C$ depending only on $\lambda, \Lambda$ and $E$, since $d_{m}<\frac{\theta^{*} R_{0}}{2}$. It is not restrictive to assume that $\eta<R_{0}^{3}\|g\|_{H^{1 / 2,1 / 4}\left(S_{T}\right)}^{2}$, since otherwise (5.14) becomes trivial. Hence, from $(5.18),(5.21)$ and $(5.22)$ we have

$$
\left(\frac{\eta}{R_{0}^{3}\|g\|_{H^{1 / 2,1 / 4}\left(S_{T}\right)}^{2}}\right)^{\frac{C}{\log \frac{R_{0}}{d_{m}}}} \geq \tilde{C}
$$

where $C$ depends on $\lambda, \Lambda$ and $E$ only and $\tilde{C}$ depends on $\lambda, \Lambda, E, M, \frac{R_{0}^{2}}{T}$ and $F$ only. Therefore (5.14) follows.

In order to prove (5.15), let us assume, with no loss of generality, that there exists $y_{0} \in \overline{\Omega_{1}}$ such that $\operatorname{dist}\left(y_{0}, \overline{\Omega_{2}}\right)=d$. Let us notice that in general $y_{0}$ need not belong to $\partial \Omega_{1}$ (see the example below Definition 5.1). Denoting $h=\operatorname{dist}\left(y_{0}, \partial \Omega_{1}\right)$, let us distinguish the following three cases:

i) $h \leq \frac{d}{2}$,

ii) $h>\frac{d}{2}, h>\frac{d_{0}}{2}$,

iii) $h>\frac{d}{2}, h \leq \frac{d_{0}}{2}$, 
where $d_{0}$ is the number introduced in Proposition 5.6.

If case i) occurs, taking $z_{0} \in \partial \Omega_{1}$ such that $\left|y_{0}-z_{0}\right|=h$, we have that $\operatorname{dist}\left(z_{0}, \overline{\Omega_{2}}\right) \geq d-h \geq \frac{d}{2}$, so that $d \leq 2 d_{m}$ and (5.15) follows from (5.14).

If case ii) occurs, let us set

$$
d_{1}=\min \left\{\frac{d}{2}, \frac{\theta d_{0}}{4}\right\}
$$

where $\theta, 0<\theta<1$, has been introduced in Proposition 5.1. We have that

$$
\begin{gathered}
\Delta_{d_{1}}\left(y_{0}\right) \subset \Omega_{1} \backslash \Omega_{2}, \\
\Delta_{\frac{\theta d_{0}}{2}}\left(y_{0}\right) \subset \Omega_{1} .
\end{gathered}
$$

By applying (5.1b) of Proposition 5.1 with $r_{1}=d_{1}, r_{2}=\frac{\theta d_{0}}{2}$, and using (5.13) and (5.25), we have

$$
\int_{0}^{T / 2} \int_{\frac{\Delta_{\frac{\theta d_{0}}{2}}\left(y_{0}\right)}{}} u_{1}^{2}(x, t) d x d t \leq C R_{0}^{3}\|g\|_{H^{1 / 2,1 / 4}\left(S_{T}\right)}^{2}\left(\frac{\eta}{R_{0}^{3}\|g\|_{H^{1 / 2,1 / 4}\left(S_{T}\right)}^{2}}\right)^{\bar{\beta} \gamma^{\prime}},
$$

where $C$ depends on $\lambda, \Lambda, E$ and $\frac{R_{0}^{2}}{T}$ only; and, moreover,

$$
\bar{\beta} \gamma^{\prime} \geq \frac{C}{\log \frac{R_{0}}{d_{1}}},
$$

where $C$ depends on $\lambda, \Lambda$ and $E$ only. By (5.26) we may apply (5.8) of Proposition 5.5 , obtaining by $(5.27),(5.28)$,

$$
d_{1} \leq \tilde{C} R_{0}\left(\frac{\eta}{R_{0}^{3}\|g\|_{H^{1 / 2,1 / 4}\left(S_{T}\right)}^{2}}\right)^{K},
$$

where $\tilde{C}>0$ and $K>0$ depend on $\lambda, \Lambda, E, M, \frac{R_{0}^{2}}{T}$ and $F$ only.

Let $\bar{\eta}=\left(\frac{\theta d_{0}}{4 \tilde{C} R_{0}}\right)^{\frac{1}{K}} R_{0}^{3}\|g\|_{H^{1 / 2,1 / 4}\left(S_{T}\right)}^{2}$. If $\eta<\bar{\eta}$, then $d_{1}<\frac{\theta d_{0}}{4}$, so that $d=2 d_{1}$ and (5.15) follows from (5.29). If, otherwise, $\eta \geq \bar{\eta}$, then (5.15) follows trivially, like what we did in (5.19).

If case iii) occurs, then $d<d_{0}$ and Proposition 5.6 applies, so that by (5.12) and (5.14) we again obtain (5.15).

Hence, by Proposition 5.3, we obtain

$$
d \leq C R_{0}\left(|\log | \log \left(\frac{R_{0}^{\frac{n-3}{2}} T^{\frac{1}{2}} \epsilon}{\|g\|_{H^{1 / 2,1 / 4}\left(S_{T}\right)}}\right)||\right)^{-K},
$$

where $C>0$ and $K>0$ depend on $\lambda, \Lambda, E, M, \frac{R_{0}^{2}}{T}$ and $F$ only. Thus we have obtained a stability estimate of log-log type. Next, by (5.30), we can find $\epsilon_{0}>0$, depending only on $\lambda, \Lambda, E, M, \frac{R_{0}^{2}}{T}$ and $F$, such that if $\epsilon \leq \epsilon_{0}$ then $d \leq d_{0}$. Therefore, by Proposition 5.6, $G$ satisfies the hypotheses of Proposition 5.4. Hence in (5.15) we may replace $\eta$ by $R_{0}^{3}\|g\|_{H^{1 / 2,1 / 4}\left(S_{T}\right)}^{2} \omega\left(\frac{R_{0}^{\frac{n-3}{2}} T^{\frac{1}{2}} \epsilon}{\|g\|_{H^{1 / 2,1 / 4}\left(S_{T}\right)}}\right)$, where $\omega$ is given by (5.7) of Proposition 5.4 (a modulus of continuity of log type), and obtain (4.10)-(4.11). 


\section{Proofs of Propositions 5.1-5.5}

Proof of Proposition 5.1. The thesis follows from (3.1.7b) of Theorem 3.1.1, with $R=\frac{\rho}{2}, r_{3}=\bar{\theta} R, \theta=\left(4 \max \left\{\sqrt{2}, \lambda_{2}\right\}\right)^{-1} \frac{\bar{\theta}}{2}$, from the trace inequality

$$
H^{2} \leq \frac{C}{T}\|u\|_{H^{2,1}(\Omega \times(0, T))}^{2},
$$

where $C$ depends on $E$ only, and from the estimate (see [LSU])

$$
\|u\|_{H^{2,1}(\Omega \times(0, T))}^{2} \leq C R_{0}^{3}\|g\|_{H^{1 / 2,1 / 4}\left(S_{T}\right)}^{2},
$$

where $C$ depends on $E$ and $\lambda$ only.

Proof of Proposition 5.2. The thesis follows from (3.2.4b) of Theorem 3.2.1, from the trace inequality

$$
\bar{H}^{2} \leq \frac{C}{T}\|u\|_{H^{2,1}(\Omega \times(0, T))}^{2},
$$

where $C$ depends on $E$ only, and from (6.2).

Proof of Proposition 5.3. Let us denote, for $i=1,2$,

$$
\mathcal{U}_{i}^{r}=\left\{x \in \overline{\Omega_{i}} \text { s.t. } \operatorname{dist}\left(x, A_{R_{0}}\right) \leq r\right\} .
$$

It is clear that

$$
\mathcal{U}_{1}^{r}=\mathcal{U}_{2}^{r}=\mathcal{U}^{r}, \quad \text { for every } r<R_{0} .
$$

From regularity estimates for solutions to parabolic equations satisfying homogeneous Neumann conditions, we have that $u_{i} \in C^{1, \alpha}\left(\overline{\Omega_{i} \backslash \mathcal{U}^{R_{0} / 8}} \times[0, T]\right), i=1,2$, and $u_{1}-u_{2} \in C^{1, \alpha}\left(\overline{\Omega_{1} \cap \Omega_{2}} \times[0, T]\right)$ for every $\alpha \in(0,1)$. Since the value of the exponent $\alpha$ is not relevant to our purposes, let us choose $\alpha=1 / 2$, obtaining

$$
\begin{gathered}
\left\|u_{i}\right\|_{C^{1,1 / 2}\left(\overline{\Omega_{i} \backslash \mathcal{U}^{R_{0} / 8}} \times[0, T]\right)} \leq C T^{-\frac{1}{2}} R_{0}^{-\frac{n-3}{2}}\|g\|_{H^{1 / 2,1 / 4}\left(S_{T}\right)}, \quad \text { for } i=1,2, \\
\left\|u_{1}-u_{2}\right\|_{C^{1,1 / 2}\left(\overline{\Omega_{1} \cap \Omega_{2}} \times[0, T]\right)} \leq C T^{-\frac{1}{2}} R_{0}^{-\frac{n-3}{2}}\|g\|_{H^{1 / 2,1 / 4}\left(S_{T}\right)},
\end{gathered}
$$

where $C>0$ depends on $\lambda, \Lambda, E, M$ and $\frac{R_{0}^{2}}{T}$ only. Now, in order to apply Theorem 3.3 .1 to $u=u_{1}-u_{2}$ in $G$, let us estimate $\left\|u_{1}-u_{2}\right\|_{H^{3 / 2,3 / 4}(\Sigma \times(0, T))}$ in terms of $\left\|u_{1}-u_{2}\right\|_{L^{2}(\Sigma \times(0, T))}$ and of the a priori data. The functions $u, u_{t}$ and $u_{t t}$ satisfy the equation

$$
u_{t}(x, t)-\operatorname{div}(\kappa(x) \nabla u(x, t))=0, \quad \text { in } G \times(0, T],
$$

and the homogeneous boundary conditions

$$
\begin{gathered}
u=0, \quad \text { on } \bar{G} \times\{0\}, \\
\kappa \nabla u \cdot \nu=0, \quad \text { on } A \times[0, T] .
\end{gathered}
$$

Hence we may apply the local boundedness estimate (see [LSU]), obtaining

$$
\begin{gathered}
\left\|u_{t}\right\|_{L^{\infty}\left(\mathcal{U}^{R_{0} / 8} \times(0, T)\right)} \leq C T^{-\frac{3}{2}} R_{0}^{-\frac{n-3}{2}}\|g\|_{H^{1 / 2,1 / 4}\left(S_{T}\right)}, \\
\left\|u_{t t}\right\|_{L^{\infty}\left(\mathcal{U}^{R_{0} / 8} \times(0, T)\right)} \leq C T^{-\frac{5}{2}} R_{0}^{-\frac{n-3}{2}}\|g\|_{H^{1 / 2,1 / 4}\left(S_{T}\right)},
\end{gathered}
$$


where $C$ depends on $\lambda, \Lambda, E$ and $\frac{R_{0}^{2}}{T}$ only. We may think at $u(\cdot, t)$ as a solution to the elliptic problem

$$
\begin{gathered}
\operatorname{div}(\kappa(x) \nabla u(x, t))=u_{t}(x, t), \quad \text { in } G, \\
\kappa \nabla u(x, t) \cdot \nu=0, \quad \text { on } A,
\end{gathered}
$$

and, similarly, we may think at $u_{t}(\cdot, t)$ as a solution to the elliptic problem

$$
\begin{gathered}
\operatorname{div}\left(\kappa(x) \nabla u_{t}(x, t)\right)=u_{t t}(x, t), \quad \text { in } G, \\
\kappa \nabla u_{t}(x, t) \cdot \nu=0, \quad \text { on } A .
\end{gathered}
$$

By $L^{p}$ regularity estimates (see $[\mathrm{GT}]$ ), by (6.6), by trace inequalities and by the immersion of $W_{p}^{2-1 / p}$ in $H^{2-1 / p}$ for $p>2$, we have

$$
\sup _{t \in[0, T]}\left(\|u(\cdot, t)\|_{H^{2-1 / p}(\Sigma)}+T\left\|u_{t}(\cdot, t)\right\|_{H^{2-1 / p}(\Sigma)}\right) \leq C T^{-\frac{1}{2}} R_{0}\|g\|_{H^{1 / 2,1 / 4}\left(S_{T}\right)},
$$

for any $p>2$, where $C$ depends on $\lambda, \Lambda, E$ and $\frac{R_{0}^{2}}{T}$ only. Therefore

$$
\|u\|_{H^{\alpha, \alpha / 2}(\Sigma \times(0, T))} \leq C R_{0}\|g\|_{H^{1 / 2,1 / 4}\left(S_{T}\right)},
$$

with $\alpha=2-1 / p>3 / 2$, where $C$ depends on $\lambda, \Lambda, E$ and $\frac{R_{0}^{2}}{T}$ only. By interpolation we have

$$
\|u\|_{H^{3 / 2,3 / 4}(\Sigma \times(0, T))} \leq C\|u\|_{H^{\alpha, \alpha / 2}(\Sigma \times(0, T))}^{1-\theta}\|u\|_{L^{2}(\Sigma \times(0, T))}^{\theta},
$$

where $\theta$ is given by $(1-\theta) \alpha=3 / 2$ (see [LioM] $)$. By (4.9), (6.7) and (6.8), choosing $p=4$ we have

$$
\|u\|_{H^{3 / 2,3 / 4}(\Sigma \times(0, T))} \leq C R_{0}\|g\|_{H^{1 / 2,1 / 4}\left(S_{T}\right)}\left(\frac{T^{1 / 2} R_{0}^{(n-3) / 2} \epsilon}{\|g\|_{H^{1 / 2,1 / 4}\left(S_{T}\right)}}\right)^{1 / 7},
$$

where $C$ depends on $\lambda, \Lambda, E$ and $\frac{R_{0}^{2}}{T}$ only. By applying Theorem 3.3.1 to $u$ and by (6.2), we have

$$
\left\|u\left(\cdot, t_{0}\right)\right\|_{L^{2}\left(\Delta_{\tilde{\theta} R_{0}}\left(P_{2}\right)\right)} \leq C R_{0}^{\frac{n}{2}}\left(\frac{\|g\|_{H^{1 / 2,1 / 4}\left(S_{T}\right)}}{T^{1 / 2} R_{0}^{(n-3) / 2}}\right)^{1-\frac{\bar{\gamma}}{7}} \epsilon^{\bar{\gamma} / 7},
$$

where $P_{2}=P_{1}-\tilde{\theta} R_{0} \nu, \nu$ denotes the outer unit normal to $\Omega$ at $P_{1}, \bar{\gamma}_{\tilde{\theta}} 0<\bar{\gamma}<1$, has been introduced in Theorem 3.3.1 and depends on $\lambda$ and $\Lambda$ only, $\tilde{\theta}, 0<\tilde{\theta}<1$, depends on $\lambda$ and $E$ only, $C \geq 1$ depends on $\lambda, \Lambda, E$ and $\frac{R_{0}^{2}}{T}$ only.

Let us prove $(5.5)-(5.6)$ when $i=1$, the case $i=2$ being analogous. Let $r \leq \tilde{\theta} R_{0}$. Let $V_{r}$ be the connected component of $\Omega_{1, r} \cap \Omega_{2, r}$ whose closure contains $\left\{x \in \Omega_{1}\right.$ s.t. $\left.\operatorname{dist}(x, \Sigma)=r\right\}$. We have

$$
\begin{gathered}
\Omega_{1} \backslash G \subset\left[\left(\Omega_{1} \backslash \Omega_{1, r}\right) \backslash G\right] \cup\left[\Omega_{1, r} \backslash V_{r}\right], \\
\partial\left(\Omega_{1, r} \backslash V_{r}\right)=\Gamma_{1, r} \cup \Gamma_{2, r},
\end{gathered}
$$

where $\Gamma_{1, r}$ is the part of boundary contained in $\partial \Omega_{1, r}$ and $\Gamma_{2, r}$ is the part contained in $\partial \Omega_{2, r} \cap \partial V_{r}$. Let us notice that

$$
\begin{gathered}
\left|\Gamma_{i, r}\right| \leq C R_{0}^{n-1}, \quad i=1,2, \\
\left|\Omega_{i} \backslash \Omega_{i, r}\right| \leq C R_{0}^{n-1} r, \quad i=1,2,
\end{gathered}
$$


where $C$ depends on $E$ and $M$ only (see Lemma 2.8 in [AlRos]). We have

$$
\int_{0}^{T / 2} \int_{\Omega_{1} \backslash G} u_{1}^{2} d x d t \leq \int_{0}^{T / 2} \int_{\left(\Omega_{1} \backslash \Omega_{1, r}\right) \backslash G} u_{1}^{2} d x d t+\int_{0}^{T / 2} \int_{\Omega_{1, r} \backslash V_{r}} u_{1}^{2} d x d t .
$$

By (6.4) and (6.11b) we have

$$
\int_{0}^{T / 2} \int_{\left(\Omega_{1} \backslash \Omega_{1, r}\right) \backslash G} u_{1}^{2} d x d t \leq C r^{\frac{1}{2}} R_{0}^{\frac{5}{2}}\|g\|_{H^{1 / 2,1 / 4}\left(S_{T}\right)}^{2},
$$

where $C$ depends on $\lambda, \Lambda, E, M$ and $\frac{R_{0}^{2}}{T}$ only. By the divergence theorem, we have

$$
\begin{gathered}
\frac{1}{2} \int_{\Omega_{1, r} \backslash V_{r}} u_{1}^{2}(x, \tau) d x \\
\leq \int_{0}^{\tau} \int_{\Gamma_{1, r}}\left|\left(u_{1} \kappa \nabla u_{1} \cdot \nu\right)\right| d s d t+\int_{0}^{\tau} \int_{\Gamma_{2, r}}\left|\left(u_{1} \kappa \nabla u_{1} \cdot \nu\right)\right| d s d t
\end{gathered}
$$

where $\nu(x)$ denotes the unit outer normal to $\Omega_{1, r}$ at $x$.

Let $x \in \Gamma_{1, r}$. We have that $d\left(x, \partial \Omega_{1}\right)=r$ and, on the other hand, that $d(x, \Sigma) \geq$ $\frac{R_{0}}{2}>r$, since $x \in \overline{\Omega_{1, r} \backslash V_{r}}$. Hence, there exists $y \in \partial \Omega_{1} \backslash \Sigma$ such that $|y-x|=$ $\operatorname{dist}\left(x, \partial \Omega_{1}\right)=r$ and moreover $\left(\kappa \nabla u_{1} \cdot \nu\right)(y, t)=0$ for every $t \in[0, T]$, where $\nu(y)$ is the unit outer normal to $\Omega_{1}$ at $y$. Since

$$
|\nu(x)-\nu(y)| \leq C \frac{r}{R_{0}}
$$

with $C$ depending only on $E$, and by (6.4) we have

$$
\left|\left(\kappa \nabla u_{1} \cdot \nu\right)(x, t)\right| \leq C T^{-\frac{1}{2}} R_{0}^{-\frac{n}{2}} r^{\frac{1}{2}}\|g\|_{H^{1 / 2,1 / 4}\left(S_{T}\right)}, \quad \text { for every } t \in[0, T],
$$

where $C$ depends on $\lambda, \Lambda, E, M$ and $\frac{R_{0}^{2}}{T}$ only.

Similarly, given $x \in \Gamma_{2, r}$, there exists $y \in \partial \Omega_{2} \backslash \Sigma$ such that $|y-x|=\operatorname{dist}\left(x, \partial \Omega_{2}\right)$ $=r$. Since $\left(\kappa \nabla u_{2} \cdot \nu\right)(y, t)=0$ for every $t \in[0, T]$, we have

$$
\left|\left(\kappa \nabla u_{1} \cdot \nu\right)(x, t)\right| \leq C\left(T^{-\frac{1}{2}} R_{0}^{-\frac{n}{2}} r^{\frac{1}{2}}\|g\|_{H^{1 / 2,1 / 4}\left(S_{T}\right)}+|\nabla u(x, t)|\right), \quad \forall t \in[0, T],
$$

where $C$ depends on $\lambda, E, M$ and $\frac{R_{0}^{2}}{T}$ only. From $(6.11)-(6.14),(6.16)-(6.17)$ and (6.4) we have

$$
\begin{gathered}
\int_{0}^{T / 2} \int_{\Omega_{1} \backslash G} u_{1}^{2} d x d t \\
\leq C R_{0}^{\frac{5}{2}}\|g\|_{H^{1 / 2,1 / 4}\left(S_{T}\right)}\left(r^{\frac{1}{2}}\|g\|_{H^{1 / 2,1 / 4}\left(S_{T}\right)}+R_{0}^{\frac{n+2}{2}}\|\nabla u\|_{L^{\infty}\left(\overline{V_{r}} \times[0, T / 2]\right)}\right),
\end{gathered}
$$

where $C$ depends on $\lambda, \Lambda, E, M$ and $\frac{R_{0}^{2}}{T}$ only. Let $(\bar{x}, \bar{t}) \in \overline{V_{r}} \times[0, T / 2]$ be such that $|\nabla u(\bar{x}, \bar{t})|=\|\nabla u\|_{L^{\infty}\left(\overline{V_{r}} \times[0, T / 2]\right)}$. Since $d\left(P_{2}, \Sigma\right)=\tilde{\theta} R_{0}$ and $d\left(P_{2}, I_{1} \cup I_{2}\right) \geq$ $(1-\tilde{\theta}) R_{0} \geq \tilde{\theta} R_{0}$, it follows that $d\left(P_{2}, \partial G\right)=\tilde{\theta} R_{0} \geq r$, so that $P_{2} \in \overline{V_{r}}$. Let $\gamma$ be an arc in $\overline{V_{r}}$ joining $\bar{x}$ to $P_{2}$. Let us define $\left\{x_{i}\right\}, i=1, \ldots, s$, as follows: $x_{1}=P_{2}$, $x_{i+1}=\gamma\left(t_{i}\right)$, where $t_{i}=\max \left\{t\right.$ s. t. $\left.\left|\gamma(t)-x_{i}\right|=2 \tilde{\theta} r\right\}$ if $\left|x_{i}-\bar{x}\right|>2 \tilde{\theta} r$; otherwise let $i=s$ and stop the process. By construction, the balls $\Delta_{\tilde{\theta} r}\left(x_{i}\right)$ are pairwise disjoint, $\left|x_{i+1}-x_{i}\right|=2 \tilde{\theta} r$, for $i=1, \ldots, s-1,\left|x_{s}-\bar{x}\right| \leq 2 \tilde{\theta} r$. Hence we have $s \leq S\left(\frac{R_{0}}{r}\right)^{n}$, with $S$ depending only on $\lambda, E$, and $M$. By iterated application 
of the three spheres inequality (3.1.7a) to $u$, with $R=\frac{r}{2}, r_{1}=\frac{\bar{\theta}}{26 \max \left\{\sqrt{2}, \lambda_{2}\right\}} r$, $r_{2}=3 r_{1}, r_{3}=13 \max \left\{\sqrt{2}, \lambda_{2}\right\} r_{1}$, over the chain of balls $\Delta_{r_{1}}\left(x_{i}\right), i=1, \ldots, s$, and by $(6.1)-(6.2)$, we have

$$
\|u(\cdot, \bar{t})\|_{L^{2}\left(\Delta_{r_{1}}(\bar{x})\right)}^{2} \leq C\left(R_{0}\left(1+\frac{T^{2}}{r^{4}}\right)\|g\|_{H^{1 / 2,1 / 4}\left(S_{T}\right)}^{2}\right)^{1-\bar{\gamma}^{s}}\|u(\cdot, \bar{t})\|_{L^{2}\left(\Delta_{r_{1}}\left(P_{2}\right)\right)}^{s},
$$

where $\bar{\gamma}, 0<\bar{\gamma}<1$, has been introduced in Theorem 3.3.1 and depends on $\lambda$ and $\Lambda$ only, $C$ depends on $\lambda, \Lambda, E$ and $\frac{R_{0}^{2}}{T}$ only. By (6.10), we have

$$
\|u(\cdot, \bar{t})\|_{L^{2}\left(\Delta_{r_{1}}(\bar{x})\right)} \leq C R_{0}\left(1+\frac{T^{2}}{r^{4}}\right)^{1-\bar{\gamma}^{s}}\|g\|_{H^{1 / 2,1 / 4}\left(S_{T}\right)}^{2} \tilde{\epsilon}^{\tilde{\epsilon}^{\frac{2}{\bar{\gamma}}} \bar{\gamma}^{s+1}}
$$

where

$$
\tilde{\epsilon}=\frac{T^{\frac{1}{2}} R_{0}^{\frac{n-3}{2}} \epsilon}{\|g\|_{H^{1 / 2,1 / 4}\left(S_{T}\right)}}
$$

and $C$ depends on $\lambda, \Lambda, E$ and $\frac{R_{0}^{2}}{T}$ only. By applying (A.2) to $u(\cdot, \bar{t})$ in $\Delta_{r_{1}}(\bar{x})$ with $\alpha=1 / 2$, by $(6.20)$ and $(6.5)$, we have

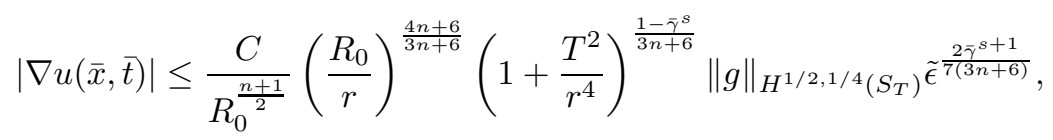

where $C$ depends on $\lambda, \Lambda, E$ and $\frac{R_{0}^{2}}{T}$ only. From $r \leq \tilde{\theta} R_{0}$, we have that $r \leq C T^{\frac{1}{2}}$, with $C$ depending on $\lambda, \Lambda, E$ and $\frac{R_{0}^{2}}{T}$ only. Therefore we can estimate

$$
1+\frac{T^{2}}{r^{4}} \leq C\left(\frac{R_{0}}{r}\right)^{4},
$$

where $C$ depends on $\lambda, \Lambda, E$ and $\frac{R_{0}^{2}}{T}$ only. By substituting (6.22) in (6.18) and by the above inequality we have

$$
\int_{0}^{T / 2} \int_{\Omega_{1} \backslash G} u_{1}^{2} d x d t \leq C R_{0}^{3}\|g\|_{H^{1 / 2,1 / 4}\left(S_{T}\right)}^{2}\left(\left(\frac{r}{R_{0}}\right)^{\frac{1}{2}}+\left(\frac{R_{0}}{r}\right)^{\frac{4 n+10}{3 n+6}}(\tilde{\epsilon})^{\frac{2 \bar{\gamma}^{s+1}}{7(3 n+6)}}\right),
$$

where $C$ depends on $\lambda, \Lambda, E, M$ and $\frac{R_{0}^{2}}{T}$ only.

Let $\bar{\mu}=\exp \left\{-\frac{7}{2}(3 n+6) \exp \left(\frac{2(S+1)|\log \bar{\gamma}|}{\bar{\theta}^{n}}\right)\right\}, \tilde{\mu}=\min \left\{\bar{\mu}, \exp \left(-\left[\frac{7}{2}(3 n+6)\right]^{2}\right)\right\}$. We have that $\tilde{\mu}<e^{-1}$, and it depends on $\lambda, \Lambda, E$ and $M$ only. It is not restrictive to assume that $\tilde{\epsilon} \leq \tilde{\mu}$, since, otherwise, (5.5) - (5.6) become trivial. Therefore, let $\tilde{\epsilon} \leq \tilde{\mu}$ and let

$$
r(\tilde{\epsilon})=R_{0}\left(\frac{2(S+1)|\log \bar{\gamma}|}{\log \left|\log \tilde{\epsilon}^{2 /(7(3 n+6))}\right|}\right)^{1 / n} .
$$

Since $r(\tilde{\epsilon})$ is increasing in $\left(0, e^{-1}\right)$ and since $r(\tilde{\mu}) \leq r(\bar{\mu})=R_{0} \tilde{\theta}$, inequality (6.23) is applicable when $r=r(\tilde{\epsilon})$ and we obtain

$$
\int_{0}^{T / 2} \int_{\Omega_{1} \backslash G} u_{1}^{2} d x d t \leq C R_{0}^{3}\|g\|_{H^{1 / 2,1 / 4}\left(S_{T}\right)}^{2}\left(\log \left|\log \tilde{\epsilon}^{2 /(7(3 n+6))}\right|\right)^{-1 / n},
$$


where $C$ depends on $\lambda, \Lambda, E, M$ and $\frac{R_{0}^{2}}{T}$ only. Since $\tilde{\epsilon} \leq \exp \left(-\left[\frac{7}{2}(3 n+6)\right]^{2}\right)$, we have that $\log \left(\frac{2}{7(3 n+6)}\right) \geq-\frac{1}{2} \log |\log \tilde{\epsilon}|$, so that

$$
\log \left|\log \tilde{\epsilon}^{\frac{2}{7(3 n+6)}}\right| \geq \frac{1}{2} \log |\log \tilde{\epsilon}|,
$$

and the thesis follows.

Proof of Proposition 5.4. Let us prove (5.5) and (5.7) when $i=1$, the case $i=2$ being analogous. By the divergence theorem we have

$$
\frac{1}{2} \int_{\Omega_{1} \backslash G} u_{1}^{2}(x, \tau) d x \leq \int_{0}^{\tau} \int_{\partial\left(\Omega_{1} \backslash G\right)} u_{1}\left(\kappa \nabla u_{1} \cdot \nu\right) d s d t, \quad \text { for every } \tau \in[0, T] .
$$

Since

$$
\partial\left(\Omega_{1} \backslash G\right) \subset\left(\partial \Omega_{1} \backslash A\right) \cup\left(\left(\partial \Omega_{2} \backslash A\right) \cap \partial G\right)
$$

and since $\kappa \nabla u_{i} \cdot \nu=0$ on $\left(\partial \Omega_{i} \backslash A\right) \times[0, T], i=1,2$, we have, by (6.26) and (6.4),

$$
\int_{0}^{T / 2} \int_{\Omega_{1} \backslash G} u_{1}^{2} d x d t \leq C R_{0}^{\frac{n+7}{2}}\|g\|_{H^{1 / 2,1 / 4}\left(S_{T}\right)}\|\nabla u\|_{L^{\infty}(\partial G \times[0, T / 2])},
$$

where $u=u_{1}-u_{2}$ and $C$ depends on $\lambda, \Lambda, E, M$ and $\frac{R_{0}^{2}}{T}$ only.

Let us introduce the following notation.

Given $z \in \mathbb{R}^{n}, \xi \in \mathbb{R}^{n},|\xi|=1, \theta>0, r>0$, we shall denote by

$$
C(z, \xi, \theta, r)=\left\{x \in \mathbb{R}^{n} \text { s. t. } \frac{(x-z) \cdot \xi}{|x-z|}>\cos \theta,|x-z|<r\right\}
$$

the intersection of the ball $\Delta_{r}(z)$ with the open cone having vertex $z$, axis in the direction $\xi$ and width $2 \theta$. Since $\partial G$ is of Lipschitz class with constants $r_{0}, L$, for any $z \in \partial G$ there exists $\xi \in \mathbb{R}^{n},|\xi|=1$, such that $C\left(z, \xi, \theta, r_{0}\right) \subset G$, where $\theta=\arctan \frac{1}{L}$.

Let $(\bar{z}, \bar{t}) \in \partial G \times[0, T / 2]$ be such that $|\nabla u(\bar{z}, \bar{t})|=\mid \nabla u \|_{L^{\infty}(\partial G \times[0, T / 2])}$. Let

$$
\begin{gathered}
\lambda_{1}=\min \left\{\frac{r_{0}}{1+\sin \theta}, \frac{r_{0}}{3 \sin \theta}, \frac{\tilde{\theta} R_{0}}{\sin \theta}\right\}, \\
\theta_{1}=\arcsin \left(\frac{\sin \theta}{l}\right), \\
w_{1}=\bar{z}+\lambda_{1} \xi, \\
\rho_{1}=\lambda_{1} \sin \theta_{1},
\end{gathered}
$$

where $l=13 \max \{\sqrt{2}, \lambda\}$ and $\tilde{\theta}$ has been introduced in Theorem 3.3.1. We have that $\Delta_{\rho_{1}}\left(w_{1}\right) \subset C\left(\bar{z}, \xi, \theta_{1}, r_{0}\right), \Delta_{l \rho_{1}}\left(w_{1}\right) \subset C\left(\bar{z}, \xi, \theta, r_{0}\right) \subset G$, so that $w_{1} \in \overline{G_{l \rho_{1}}}$. Moreover $P_{2} \in \overline{G_{l \rho_{1}}}$ since $l \rho_{1} \leq \tilde{\theta} R_{0}$, where $P_{2}$ has been introduced in Theorem 3.3.1, and $\overline{G_{l \rho_{1}}}$ is connected since $l \rho_{1} \leq \frac{r_{0}}{3}$. Arguing as in the proof of Proposition 5.3 , we obtain, by an iterated application of the three spheres inequality (3.1.7a), by $(6.1),(6.2)$ and $(6.10)$,

$$
\|u(\cdot, \bar{t})\|_{L^{2}\left(\Delta_{\rho_{1}}\left(w_{1}\right)\right)}^{2} \leq C R_{0}\|g\|_{H^{1 / 2,1 / 4}\left(S_{T}\right)}^{2} \tilde{\epsilon}^{2 \beta_{1}},
$$


where $\beta_{1}, 0<\beta_{1}<1$, depends on $\lambda, \Lambda, E, M, L$ and $\frac{R_{0}}{r_{0}}$ only and $C$ depends on $\lambda, \Lambda, E, \frac{R_{0}^{2}}{T}$ and $\frac{R_{0}}{r_{0}}$ only. Let us approach $\bar{z}$, by constructing a sequence of balls contained in $C\left(\bar{z}, \xi, \theta_{1}, r_{0}\right)$. We define, for $k \geq 2$,

$$
\begin{gathered}
w_{k}=\bar{z}+\lambda_{k} \xi, \\
\lambda_{k}=\chi \lambda_{k-1}, \\
\rho_{k}=\chi \rho_{k-1},
\end{gathered}
$$

with

$$
\chi=\frac{1-\sin \theta_{1}}{1+\sin \theta_{1}}
$$

Hence $\rho_{k}=\chi^{k-1} \rho_{1}, \lambda_{k}=\chi^{k-1} \lambda_{1}, \Delta_{\rho_{k+1}}\left(w_{k+1}\right) \subset \Delta_{3 \rho_{k}}\left(w_{k}\right) \subset \Delta_{l \rho_{k}}\left(w_{k}\right) \subset$ $C\left(\bar{z}, \xi, \theta, r_{0}\right) \subset G$. Denoting

$$
d(k)=\left|w_{k}-\bar{z}\right|-\rho_{k},
$$

we have

$$
d(k)=\chi^{k-1} d(1)
$$

with

$$
d(1)=\lambda_{1}\left(1-\sin \theta_{1}\right)
$$

For any $r, 0<r \leq d(1)$, let $k(r)$ be the smallest positive integer such that $d(k) \leq r$, that is,

$$
\frac{\left|\log \frac{r}{d(1)}\right|}{|\log \chi|} \leq k(r)-1 \leq \frac{\left|\log \frac{r}{d(1)}\right|}{|\log \chi|}+1 .
$$

By an iterated application of the three spheres inequality (3.1.7a) over the chain of balls $\Delta_{\rho_{1}}\left(w_{1}\right), \ldots, \Delta_{\rho_{k(r)}}\left(w_{k(r)}\right)$, by $(6.1),(6.2)$ and $(6.28)$, we have

$$
\|u(\cdot, \bar{t})\|_{L^{2}\left(\Delta_{\rho_{k(r)}}\left(w_{k(r)}\right)\right)}^{2} \leq C R_{0}\|g\|_{H^{1 / 2,1 / 4}\left(S_{T}\right)}^{2}\left(1+\frac{T^{2}}{\rho_{k(r)}^{4}}\right)^{1-\bar{\gamma}^{k(r)-1}} \tilde{\epsilon}^{2 \beta_{1} \bar{\gamma}^{k(r)-1}},
$$

where $\bar{\gamma}, 0<\bar{\gamma}<1$, depends on $\lambda$ and $\Lambda$ only and $C$ depends on $\lambda, \Lambda, E, \frac{R_{0}^{2}}{T}$ and $\frac{R_{0}}{r_{0}}$ only. From the definition of $\rho_{k(r)}$ we can estimate

$$
1+\frac{T^{2}}{\rho_{k(r)}^{4}} \leq \frac{C}{\chi^{4(k(r)-1)}},
$$

where $C$ depends on $\lambda, E, L, \frac{R_{0}^{2}}{T}$ and $\frac{R_{0}}{r_{0}}$ only. From (6.30), (6.31) and the interpolation inequality (A.2) with $\alpha=1 / 2$ we obtain

$$
\|\nabla u(\cdot, \bar{t})\|_{L^{\infty}\left(\Delta_{\rho_{k(r)}}\left(w_{k(r)}\right)\right)} \leq \frac{C\|g\|_{H^{1 / 2,1 / 4}\left(S_{T}\right)}}{R_{0}^{\frac{n+1}{2}}} \frac{\tilde{\epsilon}^{\beta_{2} \bar{\gamma}^{k(r)-1}}}{\chi^{\frac{4 n+10}{3 n+6}(k(r)-1)}},
$$


where $\beta_{2}=\frac{\beta_{1}}{3(n+2)}$ and $C$ depends on $\lambda, \Lambda, E, L, \frac{R_{0}^{2}}{T}$ and $\frac{R_{0}}{r_{0}}$ only. Let us consider the point $z_{r}=\bar{z}+r \xi$. We have that $z_{r} \in \Delta_{\rho_{k(r)}}\left(w_{k(r)}\right)$. From (6.32) and (6.5) we have

$$
|\nabla u(\bar{z}, \bar{t})| \leq \frac{C\|g\|_{H^{1 / 2,1 / 4}\left(S_{T}\right)}}{R_{0}^{\frac{n+1}{2}}}\left(\left(\frac{r}{R_{0}}\right)^{\frac{1}{2}}+\frac{\tilde{\epsilon}^{\beta_{2} \bar{\gamma}^{k(r)-1}}}{\chi^{\frac{4 n+10}{3 n+6}(k(r)-1)}}\right),
$$

where $C$ depends on $\lambda, \Lambda, E, M, L, \frac{R_{0}^{2}}{T}$ and $\frac{R_{0}}{r_{0}}$ only. Let

$$
r(\tilde{\epsilon})=d(1)\left|\log \tilde{\epsilon}^{\beta_{2}}\right|^{-B}
$$

with

$$
B=\frac{|\log \chi|}{2|\log \bar{\gamma}|}
$$

Let $\tilde{\mu}=\exp \left(-\beta_{2}^{-1}\right)$. We have that $r(\tilde{\mu})=d(1)$ and $r(\tilde{\epsilon}) \leq d(1)$ for any $\tilde{\epsilon}, 0<\tilde{\epsilon} \leq \tilde{\mu}$. It is not restrictive to assume that $0<\tilde{\epsilon} \leq \tilde{\mu}$ since, otherwise, (5.5), (5.7) become trivial. Therefore inequality (6.33) is applicable when $r=r(\tilde{\epsilon})$. Recalling (6.27) and (6.29), we obtain

$$
\int_{0}^{T / 2} \int_{\Omega_{1} \backslash G} u_{1}^{2} d x d t \leq C R_{0}^{3}\|g\|_{H^{1 / 2,1 / 4}\left(S_{T}\right)}^{2}\left|\log \tilde{\epsilon}^{\beta_{2}}\right|^{-B},
$$

where $C$ depends on $\lambda, \Lambda, E, M, L, \frac{R_{0}^{2}}{T}$ and $\frac{R_{0}}{r_{0}}$ only. Therefore (5.5) and (5.7) follow.

Let us precede the proof of Proposition 5.5 with the following lemma, which states a Caccioppoli-type inequality for solutions to parabolic equations vanishing at time $t=0$.

Lemma 6.1. Let $u \in H^{2,1}\left(\Delta_{r} \times(0, T)\right.$ be a solution to (4.1a)-(4.1b), where $\kappa$ satisfies (4.8a). We have

$$
\int_{0}^{\tau} \int_{\Delta_{s}}|\nabla u|^{2} d x d t \leq \frac{c \lambda^{4}}{(r-s)^{2}} \int_{0}^{\tau} \int_{\Delta_{r}} u^{2} d x d t
$$

for every $\tau \in[0, T]$ and for every $s \in(0, r)$.

Proof of Lemma 6.1. Let $\eta \in C_{0}^{\infty}\left(\Delta_{r}\right)$ be such that $\eta \equiv 1$ on $\Delta_{s},|\nabla \eta| \leq \frac{c}{r-s}$. By using as test function $\varphi=\eta^{2} u$, we get

$$
\int_{0}^{\tau} \int_{\Delta_{r}} \eta^{2} \kappa \nabla u \cdot \nabla u d x d t+\int_{0}^{\tau} \int_{\Delta_{r}} 2 \eta u \kappa \nabla u \cdot \nabla \eta d x d t=\frac{1}{2} \int_{\Delta_{r}} \eta^{2} u^{2}(x, \tau) d x \leq 0 .
$$

Hence

$$
\begin{gathered}
\lambda^{-1} \int_{0}^{\tau} \int_{\Delta_{r}} \eta^{2}(x)|\nabla u|^{2} d x d t \\
\leq 2 \lambda\left(\int_{0}^{\tau} \int_{\Delta_{r}} \eta^{2}|\nabla u|^{2} d x d t\right)^{1 / 2}\left(\int_{0}^{\tau} \int_{\Delta_{r}} u^{2}|\nabla \eta|^{2} d x d t\right)^{1 / 2} .
\end{gathered}
$$

Finally, we have

$$
\int_{0}^{\tau} \int_{\Delta_{s}}|\nabla u|^{2} d x d t \leq \int_{0}^{\tau} \int_{\Delta_{r}} \eta^{2}|\nabla u|^{2} d x d t \leq \frac{4 \lambda^{4} c^{2}}{(r-s)^{2}} \int_{0}^{\tau} \int_{\Delta_{r}} u^{2} d x d t,
$$

and the thesis follows. 
Proof of Proposition 5.5. Arguing similarly to the proof of Proposition 4.3 in AlBRV], we can prove the following trace inequality. Let $l=13 \max \{\sqrt{2}, \lambda\}$. For every $d \leq \frac{R_{0}}{8 l \sqrt{1+E^{2}}}$ and for every $t \in[0, T]$ we have

$$
\int_{\partial \Omega} u_{x_{i}}^{2}(x, t) d s \leq C\left(d \int_{\Omega}\left|\nabla u_{x_{i}}(x, t)\right|^{2} d x+\frac{1}{d} \int_{\Omega_{l d}} u_{x_{i}}^{2}(x, t) d x\right),
$$

and, integrating (6.36) over $[0, T / 2]$,

$$
\int_{0}^{T / 2} \int_{\partial \Omega} u_{x_{i}}^{2} d s d t \leq C\left(d \int_{0}^{T / 2} \int_{\Omega}\left|D^{2} u\right|^{2} d x d t+\frac{1}{d} \int_{0}^{T / 2} \int_{\Omega_{l d}} u_{x_{i}}^{2} d x d t\right)
$$

for $i=1, \ldots, n$, where $C$ depends on $E$ and $M$ only. Let $\rho>0$ and $x_{0} \in \Omega_{\rho}$. Let us denote

$$
\epsilon^{2}=\int_{0}^{T / 2} \int_{\Delta_{\rho}\left(x_{0}\right)} u^{2} d x d t
$$

Let $d \leq d_{0}$, with $d_{0}=\min \left\{\frac{R_{0}}{8 l \sqrt{1+E^{2}}}, \frac{\bar{\theta} \rho}{2 l}, \frac{R_{0}}{3 l}\right\}$, where $\bar{\theta}$ has been introduced in Proposition 3.5. Let us notice that $\frac{R_{0}}{d_{0}}$ depends on $\lambda, \Lambda, E, \frac{R_{0}^{2}}{T}$ and $\frac{R_{0}}{\rho}$ only. Moreover $x_{0} \in \Omega_{l d}$ and $\Omega_{l d}$ is connected. Let $x \in \Omega_{l d}$. By iterating the three cylinders inequality $(3.1 .7 \mathrm{~b})$ with $r_{1}=d, r_{2}=3 d, r_{3}=l d$ over a chain of cylinders $\Delta_{d}\left(x_{k}\right) \times[0, T / 2]$, with $x_{k}, k=1, \ldots, N_{d}$, points of a path in $\Omega_{l d}$ joining $x$ to $x_{0}$, and by (6.1), we obtain

$$
\int_{0}^{T / 2} \int_{\Delta_{d}(x)} u^{2} d x d t \leq C\left(\left(1+\frac{T^{2}}{d^{4}}\right)\|u\|_{H^{2,1}(\Omega \times(0, T))}^{2}\right)^{1-\bar{\gamma}^{N_{d}}} \epsilon^{2 \bar{\gamma}^{N_{d}}},
$$

where $\bar{\gamma}, 0<\bar{\gamma}<1$, depends on $\lambda$ and $\Lambda$ only, $C$ depends on $\lambda, \Lambda$ and $\frac{R_{0}^{2}}{T}$ only, and $N_{d} \leq \frac{M R_{0}^{n}}{\omega_{n} d^{n}}$. Let us cover $\Omega_{l d}$ with internally nonoverlapping closed cubes $\left\{Q_{j}\right\}_{j=1, \ldots N_{d}^{\prime}}$, of side $d /(2 \sqrt{n})$. For every $j=1, \ldots, N_{d}^{\prime}$, let $x^{j} \in \Omega_{l d} \cap Q_{j}$, so that $Q_{j} \subset \Delta_{d / 2}\left(x_{j}\right)$. By Lemma 6.1 we have, for every $i=1, \ldots, n$,

$$
\begin{gathered}
\int_{0}^{T / 2} \int_{\Omega_{l d}} u_{x_{i}}^{2} d x d t \leq \sum_{j=1}^{N_{d}^{\prime}} \int_{0}^{T / 2} \int_{Q_{j}} u_{x_{i}}^{2} d x d t \\
\leq \sum_{j=1}^{N_{d}^{\prime}} \int_{0}^{T / 2} \int_{\Delta_{d / 2}\left(x_{j}\right)} u_{x_{i}}^{2} d x d t \leq \frac{C}{d^{2}} N_{d}^{\prime} \int_{0}^{T / 2} \int_{\Delta_{d / 2}\left(x_{j}\right)} u^{2} d x d t,
\end{gathered}
$$

where $N_{d}^{\prime} \leq C \frac{R_{0}^{n}}{d^{n}}$, with $C$ depending only on $M$. Let $\alpha=\frac{\epsilon^{2}}{e^{2}\|u\|_{H^{2,1}(\Omega \times(0, T))}}$. From $(6.37)-(6.39)$ we have

$$
\int_{0}^{T / 2} \int_{\partial \Omega}|\nabla u|^{2} d s d t \leq \frac{C}{R_{0}^{3}}\|u\|_{H^{2,1}(\Omega \times(0, T))}^{2}\left(\frac{d}{R_{0}}+\left(\frac{R_{0}}{d}\right)^{n+7} \alpha^{\bar{\gamma}^{N_{d}}}\right),
$$

where $C$ depends on $\lambda, \Lambda, M, E$ and $\frac{R_{0}^{2}}{T}$ only. Let

$$
\alpha_{0}=\exp \left(-\exp \left(\frac{2 M|\log \bar{\gamma}|}{\omega_{n}}\left(\frac{R_{0}}{d_{0}}\right)^{n}\right)\right)<1 .
$$


We have that $\alpha_{0}$ depends on $\lambda, \Lambda, E, M, \frac{R_{0}^{2}}{T}$ and $\frac{R_{0}}{\rho}$ only. If $\alpha \leq \alpha_{0}$ we choose $d=d_{\alpha}$, where

$$
d_{\alpha}=R_{0}\left(\frac{2 M|\log \bar{\gamma}|}{\omega_{n} \log |\log \alpha|}\right)^{1 / n} .
$$

Then $d_{\alpha} \leq d\left(\alpha_{0}\right)=d_{0}$, and from (6.40) we have

$$
\int_{0}^{T / 2} \int_{\partial \Omega}|\nabla u|^{2} d s d t \leq \frac{C}{R_{0}^{3}}\|u\|_{H^{2,1}(\Omega \times(0, T))}^{2}(\log |\log \alpha|)^{-1 / n},
$$

where $C$ depends on $\lambda, \Lambda, E, M$ and $\frac{R_{0}^{2}}{T}$ only. If $\alpha>\alpha_{0}$, from (6.40), using the fact that $\alpha \leq 1$, and replacing $d$ by $d_{0}$, we obtain

$$
\int_{0}^{T / 2} \int_{\partial \Omega}|\nabla u|^{2} d s d t \leq \frac{C}{R_{0}^{3}}\|u\|_{H^{2,1}(\Omega \times(0, T))}^{2}\left(\frac{R_{0}}{d_{0}}\right)^{n+7} \frac{\alpha}{\alpha_{0}},
$$

where $C$ depends on $\lambda, \Lambda, E, M$ and $\frac{R_{0}^{2}}{T}$ only.

Since $\alpha \leq e^{-2}$ we have $\alpha(\log |\log \alpha|)^{1 / n} \leq e^{-2}(\log 2)^{1 / n}$, so that from (6.41)(6.42) we have

$$
\int_{0}^{T / 2} \int_{\partial \Omega} g^{2} d s d t \leq \frac{C}{\alpha_{0} R_{0}^{3}}\|u\|_{H^{2,1}(\Omega \times(0, T))}^{2}\left(\frac{R_{0}}{d_{0}}\right)^{n+7}(\log |\log \alpha|)^{-1 / n},
$$

where $C$ depends on $\lambda, \Lambda, E, M$ and $\frac{R_{0}^{2}}{T}$ only. From (6.43) and from

$$
C_{1} R_{0}^{3}\|g\|_{H^{1 / 2,1 / 4}\left(S_{T}\right)}^{2} \leq\|u\|_{H^{2,1}(\Omega \times(0, T))}^{2} \leq C_{2} R_{0}^{3}\|g\|_{H^{1 / 2,1 / 4}\left(S_{T}\right)}^{2},
$$

where $C_{1}, C_{2}$ depend on $\lambda, E$ only, we have

$$
\epsilon^{2} \geq e^{2} R_{0}^{3} \exp \left(-\exp \left(\frac{C}{\alpha_{0}}\left(\frac{R_{0}}{d_{0}}\right)^{n+7} F^{2}\right)^{n}\right)\|g\|_{H^{1 / 2,1 / 4}\left(S_{T}\right)}^{2},
$$

where $C$ depends on $\lambda, \Lambda, E, M$ and $\frac{R_{0}^{2}}{T}$ only. Therefore (5.8) follows with the stated dependence.

\section{Appendix A. Interpolation and Trace Inequalities}

Given an interval $I$ in $\mathbb{R}$, we have

$$
\|f\|_{L^{\infty}(I)} \leq c\left(|I|\left\|f^{\prime}\right\|_{L^{2}(I)}^{2}+\frac{1}{|I|}\|f\|_{L^{2}(I)}^{2}\right)^{1 / 2},
$$

where $|I|$ denotes the length of the interval $I$.

Given a function $v$ of class $C^{1,1}$ in $\Delta_{\rho} \subset \mathbb{R}^{n}$, for every $\alpha \in(0,1]$ we have

$$
\begin{gathered}
\|\nabla v\|_{L^{\infty}\left(\Delta_{\rho}\right) \leq} \frac{c}{\rho^{1+\frac{n \alpha}{(n+2)(1+\alpha)}}}\|v\|_{C^{1, \alpha}}^{\frac{n \alpha+n+2}{(n+2) \alpha}}\|v\|_{L^{2}}^{\frac{2 \alpha}{(n+2)(1+\alpha)}} . \\
\left(\frac{1}{\rho^{n-2}} \int_{\Delta_{\rho}}|\nabla f|^{2} d x\right)^{1 / 2} \\
\leq C\left(\left(\rho^{1+\alpha}|\nabla f|_{\alpha, \rho^{\prime}}\right)^{\frac{1}{1+\alpha}}\left(\frac{1}{\rho^{n}} \int_{\Delta_{\rho}} f^{2} d x\right)^{\frac{\alpha}{2(1+\alpha)}}+\left(\frac{1}{\rho^{n}} \int_{\Delta_{\rho}} f^{2} d x\right)^{1 / 2}\right),
\end{gathered}
$$

where $\rho<\rho^{\prime}<2 \rho, 0<\alpha<1$ and $C$ depends on $\alpha$ only. 
For every $\rho<r$, we have

$$
\begin{aligned}
& \int_{\Delta_{\rho}}|F(x, 0)|^{2} d x \leq c\left(\frac{r}{r^{2}-\rho^{2}} \int_{B_{r}^{+}}|F(x, y)|^{2} d x d y+r \int_{B_{r}^{+}}\left|F_{y}(x, y)\right|^{2} d x d y\right), \\
& \quad \int_{B_{r}^{+}}|F(x, y)|^{2} d x d y \leq c\left(r \int_{\Delta_{r}}|F(x, 0)|^{2} d x+r^{2} \int_{B_{r}^{+}}\left|F_{y}(x, y)\right|^{2} d x d y\right) .
\end{aligned}
$$

\section{ACKNOWLEDGMENT}

The authors wish to thank Giovanni Alessandrini for reading the first draft of this paper and for useful suggestions.

\section{REFERENCES}

[AE] V. Adolfsson and L. Escauriaza, $C^{1, \alpha}$ domains and unique continuation at the boundary, Comm. Pure Appl. Math. L (1997), 935-969. MR 98m:31003

[Al] G. Alessandrini, Examples of instability in inverse boundary-value problems, Inverse Problems 13 (1997), 887-897. CMP 97:16

[AlBRV] G. Alessandrini, E. Beretta, E. Rosset, and S. Vessella, Optimal stability for inverse elliptic boundary value problems with unknown boundaries, Ann. Scuola Norm. Sup. Pisa Cl. Sci. (4) XXIX (2000), 755-806. CMP 2001:10

[AlR] G. Alessandrini and L. Rondi, Optimal stability for the inverse problem of multiple cavities, J. Differential Equations (to appear).

[AlRos] G. Alessandrini and E. Rosset, The inverse conductivity problem with one measurement: bounds on the size of the unknown object, SIAM J. Appl. Math. 58 (1998), 1060-1071. MR 99j:35227

[BryC1] K. Bryan and L. F. Caudill, Jr., Uniqueness for a boundary identification problem in thermal imaging, Proc. Third Mississippi State Conf. Differential Equations and Computational Simulations (J. Graef et al., eds.), Electron. J. Differ. Eq. Conf. 1 (1998), 23-39. MR 2000g:35226

[BryC2] K. Bryan and L. F. Caudill, Jr., Stability and reconstruction for an inverse problem for the heat equation, Inverse Problems 14 (1998), 1429-1453. MR 99m:35258

[C] B. Canuto, Une contribution à l'etude de quelques problèmes inverses pour des equations paraboliques, Thèse de Doctorat de l'Université de Versailles (1999).

[CH] R. Courant and D. Hilbert, Methods of mathematical physics, Vol. I, Interscience, New York, 1962. MR 16:426a (earlier ed.)

[GL] N. Garofalo and F. Lin, Monotonicity properties of variational integrals, $A_{p}$ weights and unique continuation, Indiana Univ. Math. J. 35 (1986), 245-268. MR 88b:35059

[GT] D. Gilbarg and N. S. Trudinger, Elliptic partial differential equations of second order, Springer, New York, 1983. MR 86c:35035

[Is1] V. Isakov, Inverse problems for partial differential equations, Springer, New York, 1998. MR 99b:35211

[Is2] V. Isakov, Some inverse problems for the diffusion equation, Inverse Problems 15 (1999), 3-10. MR 99j:35231

[ItY] S. Ito and H. Yamabe, A unique continuation theorem for solutions of a parabolic differential equation, J. Math. Soc. Japan 10 (1958), 314-321. MR 20:5958

[K] I. Kukavica, Quantitative uniqueness for second-order elliptic operators, Duke Math. J. 91 (1998), 225-240. MR 99a:353046

[LSU] O. A. Ladyzhenskaja, V. A. Solonnikov, and N. N. Ural'ceva, Linear and quasilinear equations of parabolic type, Translations of Math. Monographs 23, Amer. Math. Soc., Providence, 1968. MR 39:3159

[Lan] E. M. Landis, A three-spheres theorem, Soviet Math. Dokl. 4 (1963), 76-78. MR 27:443

[Lan-O] E. M. Landis and O. A. Oleinik, Generalized analiticity and some related properties of solutions of elliptic and parabolic equations, Russian Math. Surveys 29 (1974), no. 2, 195-212. MR 53:6089 
[LavRS] M. M. Lavrent'ev, V. G. Romanov, and S. P. Shishatskii, Ill-posed problems of mathematical physics and analysis, Translations of Math. Monographs 64, Amer. Math. Soc., Providence, 1986. MR 87g:65003

[LeP] M. Lees and M. H. Protter, Unique continuation for parabolic differential equations and inequalities, Duke Math. J. 28 (1961), 369-382. MR 25:4254

[Li] F. H. Lin, A uniqueness theorem for parabolic equations, Comm. Pure Appl. Math. XLIII (1990), 127-136. MR 90j:35106

[LioM] J. L. Lions and E. Magenes, Non-homogeneous boundary value problems and applications II, Springer, New York, 1972. MR 50:2671

[M] C. B. Morrey, Jr., Multiple integrals in the calculus of variations, Springer, New York, 1966. MR 34:2380

[P] M. H. Protter, Properties of solutions of parabolic equations and inequalities, Canad. J. Math 13 (1961), 331-345. MR 27:3443

[V] S. Vessella, Stability estimates in an inverse problem for a three-dimensional heat equation, SIAM J. Math. Anal. 28 (1997), 1354-1370. MR 99d:35178

[Y] H. Yamabe, A unique continuation theorem of a diffusion equation, Annals of Mathematics 69 (1959), 462-466. MR 21:206

Laboratoire de Maths Appliquées, Université de Versailles-St. Quentin, Bâtiment Fermat 45, Avenue des États-Unis, 78035 Versailles Cedex, France

E-mail address: canuto@math.uvsq.fr

Dipartimento di Scienze Matematiche, Università degli Studi di Trieste, Via Valerio 12/1, 34100 TRIESTE, Italy

E-mail address: rossedi@univ.trieste.it

Dipartimento di Matematica per le Decisioni (Dimad), Università degli Studi di Firenze, Via C. Lombroso 6/17, 50134 Firenze, Italy

E-mail address: vessella@ds.unifi.it 